\title{
PLASTICIDAD DE MATERIALES POLICRISTALINOS CON POROSIDAD PRESURIZADA
}

\author{
Tesis de Maestría \\ JUAN E. RAMOS NERVI \\ Presentada ante la Facultad de Ingeniería de la \\ Universidad Nacional de La Plata \\ como requisito para la obtención del grado académico de

\section{MAGÍSTER EN INGENIERÍA}

Dirección de tesis:

\author{
Dr. Ing. Martín I. Idiart
}

Jurado de tesis:

Dr. Javier Signorelli, Dr. Adrián Cisilino, Dra. Alicia Denis.

21 de diciembre de 2015 



\section{Resumen}

Tanto los materiales utilizados como internos en reactores nucleares de potencia como las pastillas combustibles son materiales heterogéneos policristalinos que experimentan cambios a nivel microscópico durante su irradiación como la nucleación de microporosidad y su evolución dictadas por la fracción volumétrica de poros y su presión interna. Dichos cambios determinan su comportamiento mecánico macroscópico limitando la vida del componente o, en el caso de la pastilla, la acción que genera sobre la vaina que las contiene comprometiendo de esta forma su integridad.

Los materiales policristalinos son agregados de granos compuestos de cristales simples orientados aleatoriamente y/o con una orientación preferencial asociada al proceso de deformación al que se ven sometidos. Su comportamiento constitutivo es dictado en gran medida por la morfología, la orientación cristalina, la respuesta de cada grano individual o cristal simple que constituye el agregado y la microporosidad. Para estimar la anisotropía en su comportamiento plástico cuando se someten a grandes deformaciones es necesario relacionar la respuesta macroscópica con las propiedades microscópicas. Comúnmente la respuesta de estos materiales es idealizada como elásticamente rígida y perfectamente plástica sin endurecimiento. El problema anterior se reduce a encontrar la superficie de fluencia del policristal dada la superficie de fluencia del cristal simple y la estadística tanto morfológica como de distribución de orientaciones de los granos y cavidades. Debido a su inherente aleatoriedad microestructural, los sólidos policristalinos no muestran una única respuesta, sino una gama de respuestas. En este trabajo se derivan cotas para la gama de posibles respuestas. Para ello, se hace uso del método de medio lineal de comparación propuesto por Idiart \& Ponte Castañeda 2007a-b (Proc. R. Soc. Lond. 463, 907-943). El efecto de la presión interna se introduce en las cotas a través de un cambio adecuado de variables siguiendo el trabajo de Vincent et al. 2009 (Int. J. Solids Struct. 46, 480-506). El método se aplica a distintos sistemas materiales a fin de explorar el efecto simultáneo de la simetría cristalina y el nivel de presión interna en la resistencia plástica global de sólidos policristalinos. 



\begin{abstract}
Reactor internals and fuel pellets used in Nuclear Power Plants are polycrystalline heterogeneous materials that exhibit irradiation-induced microestructural changes such as micro - porosity nucleation and evolution dictated by the pore volumen fraction and their internal preassure. These affects the operational life limit of structural components and - in case of fuel pellets - can compromise the cladding integrity.

Polycrystalline materials are aggregates of randomly oriented single-crystal grains. Their elastoplastic deformation is to a great extent dictated by the morphology, lattice orientation, and elastoplastic response of each individual single-crystal grain composing the aggregate, the porosity, and the internal gas pressure. Relating the macroscopic response with the microscopic properties is necessary to estimate the deformation-induced plastic anisotropy that develops in these materials when subjected to large deformations. Very often the response of these materials is idealized as elastically rigid and plastically non-hardening. Within this so-called rigid-perfectly plastic model the above problem reduces to finding the macroscopic yield surface of the polycrystal given the yield surface at the single-crystal level and the statistics of the morphology and orientation distributions of the grains and cavities. Due to their inherent microstructural randomness, cognate polycrystalline solids do not exhibit a single response but a — hopefully narrow — range of responses. In this work we derive bounds for the range of possible responses.

To that end, we make use of the of a comparison-medium method proposed by Idiart \& Ponte Castañeda 2007a-b (Proc. R. Soc. Lond. 463, 907-943). The effect of internal pressure is then introduced in the bounds via a suitable change of variables following the work of Vincent et al. 2009 (Int. J. Solids Struct. 46, 480-506). The method is applied to various material systems in order to explore the simultaneous effect of crystallographic symmetry and internal pressure level on the overall plastic strength of polycrystalline solids.
\end{abstract}





\section{Prefacio}

Nucleoeléctrica Argentina S.A. (N.A.-S.A.) es la operadora de las Centrales Nucleares de Potencia en la República Argentina. Se encuentra actualmente realizando la extensión de vida de la Central Nuclear Embalse, realizando estudios previos en la Central Nuclear Juan D. Péron (Atucha I) y planea la construcción de otras centrales. En este contexto, se busca mejorar el conocimiento del comportamiento de los materiales nucleares y su evolución microestructural con la irradiación, ya que éste posee gran impacto en aspectos regulatorios, de integridad estructural y seguridad nuclear. Para ello es necesario desarrollar modelos micromecánicos que dan cuenta de dichos efectos; por ejemplo considerando la distribución de porosidad y otros fenómenos a nivel microscópico. Estos modelos se desarrollan en un contexto general para luego ajustarse a aplicaciones específicas integrándose con otros modelos macroscópicos y fenomenológicos más simples, reduciendo su conservativismo y extendiendo su rango de uso. Por ejemplo, para las aleaciones de circonio los modelos micromecánicos permiten estimar parámetros macroscópicos de programas como el $\mathrm{MACCACO}^{1}$ que se utiliza para modelar la deformación de los tubos de presión; para los aceros y aleaciones base níquel, permiten estimar parámetros e incorporar complejas leyes constitutivas a nivel cristal simple para la predicción de su comportamiento mecánico a fin de vida y durante su vida extendida; en el caso de las pastillas combustibles, se utilizan — entre varias aplicaciones - para la descripción de su comportamiento plástico en función de la microestructura permitiendo el acoplamiento con códigos de combustible multifísicos como el DIONISIO ${ }^{1}$, BACO $^{1}$ o el TRANSURANUS $^{2}$ que modelan la deformación de la pastilla, su eventual acción sobre la vaina.

Los resultados de este trabajo han sido parcialmente presentados en las siguientes publicaciones:

- Ramos Nervi J.E. \& Idiart M.I. 2015. Bounding the plastic strength of polycrystalline voided solids by linear-comparison homogenization techniques. Proc. R. Soc. A 471: 20150380.

\footnotetext{
${ }^{1}$ Desarrollado por la Comisión Nacional de Energía Atómica -CNEA.

${ }^{2}$ Desarrollado en la Unión Europea y adoptado por NA-SA.
} 
- Idiart M.I. \& Ramos Nervi J.E. 2014. Bounds on the hydrostatic plastic strength of voided polycrystals and implications for linear-comparison homogenization techniques. Comptes Rendus Mecanique 342, 25-31.

Agradezco el apoyo recibido durante estos años por parte de la empresa para llevar a cabo este trabajo, en particular a los Ings. Schroeter, F., Antonaccio, C., Moliterno, G. y al Lic. Sidelnik, J.; Ing. Antunez, J.; Ing. Ovando, L. Todos ellos contribuyeron apoyando este proyecto generando una visión de empresa sustentada en el conocimiento para el desarrollo del plan nuclear argentino. En la Comisión Nacional de Energía Atómica, por las discusiones de las aplicaciones de los modelos: Dr. Chocrón, M.; Dra. Fortis, A.M.; especialmente a la Dra. Bozzano, P. Al jurado de esta tesis por sus valiosos aportes durante su etapa final: Dr. Signorelli, J.; Dra. Denis, A.; Dr. Cisilino, A.; Dr. Chocrón, M. y a mis compañeros de la UNLP por su consideración y discusiones: Villar, J.; Bottero, C.; Zappitelli, M.P. y finalmente al Dr. Idiart, M.I. por su paciencia y brindarme la oportunidad de aprender.

Parte del trabajo de tesis se realizó en el marco del PROYECTO DE VINCULACIÓN y TRANFERENCIA TECNOLÓGICA "CAPACIDADES UNIVERSITARIAS PARA EL DESARROLLO PRODUCTIVO, Amílcar Oscar Herrera: ASPECTOS MECÁNICOS DE ALEACIONES ALTERNATIVAS PARA CANALES DE REFRIGERACIÓN DE CENTRALES NUCLEARES", financiado por el Ministerio de Educación de la Nación.

En el Departamento de Mecánica de la Gerencia de Ingeniería de NA-SA se desarrollaron en el transcurso de este trabajo los siguientes programas aplicando los conocimientos adquiridos:

- Modelo de transporte de deuterio en tubos de presión. TRADE v 0.1.2014.

- Modelo micromecánico termoelástico anisótropo. TELAGEN v 0.1.2015.

- Modelo de mecánica de fractura lineal elástica. FLEPLA v 0.1.2016. 


\section{Indice}

1. Introducción 1

2. Modelo de policristales porosos presurizados 13

2.1. Modelo del sólido poroso policristalino . . . . . . . . . . . . . . . 13

2.2. Algunos sistemas materiales . . . . . . . . . . . . . . . . . . 16

2.3. Efecto de la presión interna en la superficie de fluencia del policristal . . . 18

3. Cotas para superficies de fluencia de policristales porosos 21

3.1. Métodos basados en medios lineales de comparación . . . . . . . . . . . 21

3.1.1. Relajaciones . . . . . . . . . . . . . . . . . . . . 26

3.2. Cotas de resistencia plástica hidrostática de policristales porosos isótropos 27

3.2.1. Aplicación a sólidos cúbicos policristalinos . . . . . . . . . . . 27

3.2.2. Aplicación a sólidos hexagonales policristalinos . . . . . . . . . 30

3.2.3. Resultados para sistemas materiales específicos . . . . . . . . . . 31

4. Resultados para policristales isótropos cúbicos y hexagonales 35

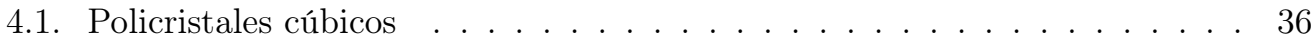

4.2. Policristales hexagonales . . . . . . . . . . . . . . . . . . . 41

4.3. Policristales con sistemas de deslizamiento deficientes . . . . . . . . . . . . 42

4.4. Cotas de resistencia hidrostática . . . . . . . . . . . . . . . . . 45

5. Conclusiones de la tesis $\quad 49$

A. Aspectos numéricos

B. Cotas de resistencia hidrostática para sólidos policristalinos con siste-

mas deficientes $\quad 57$

$\begin{array}{ll}\text { Bibliografía } & 60\end{array}$ 



\section{Capítulo 1}

\section{Introducción}

La mayoría de los materiales ingenieriles son intrínsecamente heterogéneos. Entre los de aplicación nuclear se encuentran los metales internos del reactor como aceros austeníticos, aleaciones base níquel, aleaciones de circonio (véase figura 1.1 a-b) y los cerámicos como las pastillas combustibles (véase figura 1.1 a). Estos materiales son agregados policristalinos donde los tamaños de grano son típicamente del orden de los micrones. Durante la irradiación en el reactor las propiedades de sus constituyentes y su distribución espacial (microestructura) evolucionan, por ejemplo, presentando distribuciones de poros orientadas aleatoriamente que son del tamaño de algunos micrones. En la mayoría de los problemas de integridad estructural y en particular en las aplicaciones mencionadas anteriormente, la longitud de la escala en consideración es del orden del milímetro o mayor mientras que el tamaño de las heterogeneidades consideradas (es decir, partículas, poros, granos, etc.) es mucho más pequeña. Por lo tanto, la relación entre la talla típica de las heterogeneidades respecto del tamaño de los componentes es muy pequeña; es decir la separación en la longitud de escalas es de gran importancia. En este caso es común reemplazar el material heterogéneo por uno equivalente homogéneo. Dicho material equivalente es caracterizado por cierto comportamiento macroscópico homogeneizado el cual debe representar con precisión el comportamiento del material heterogéneo. La respuesta macroscópica del compuesto depende su microestructura. En la mayoría de los compuestos es extremadamente compleja y únicamente se puede realizar una descripción estadística parcial de la misma. En consecuencia, la respuesta macroscópica no puede ser 
determinada en general de manera exacta, pero puede ser estimada. Para ello se utilizan los métodos de homogeneización que permiten conectar las escalas microscópicas y macroscópicas.

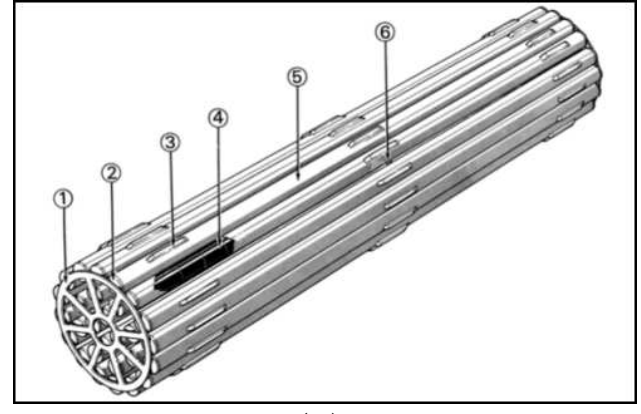

(a)

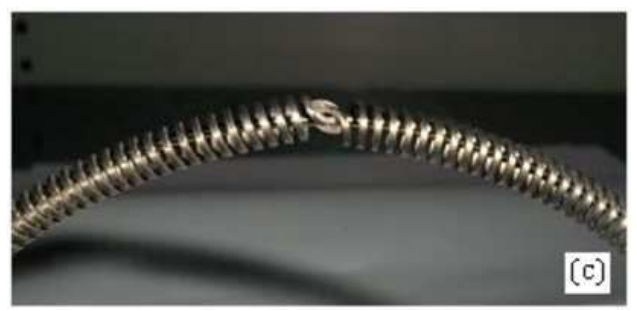

(b)

Figura 1.1: Ejemplos de materiales policristalinos utilizados en el núcleo de centrales nucleares de potencia CANDU600: (a) elemento combustible. Las pastillas combustibles cerámicas de $U_{2}$ se alojan en las vainas de Zry4, las mismas están unidas por separadores de aleaciones base níquel y poseen partes de acero inoxidable; (b) anillos espaciadores de inconel X-750 con espiras centrales de una aleación de circonio, separan el tubo de presión del tubo de calandria.

Entre los cambios microestructurales producidos por irradiación, podemos destacar la nucleación y evolución de microcavidades presurizadas a distintas escalas; la segregación, precipitación y/o disolución de partículas de segundas fases; el cambio en la relación de aspecto de las celdas cristalinas; en el caso de las pastillas de alto quemado, la recristalización dinámica de la zona periférica, nucleación y crecimiento de fases porosas, etc. A continuación se describen brevemente distintos aspectos microestructurales de las aplicaciones ingenieriles mencionadas en la industria nuclear.

La pastilla combustible es un sólido cerámico policristalino cúbico iónico que durante el quemado en el reactor presenta una nucleación de porosidad a distintas escalas. En la figura 1.2 se muestra para el caso de quemados típicos de un $\mathrm{PWR}^{1}$ las diferentes poblaciones de poros que se forman entre los granos del material y dentro de los mismos. Este fenómeno ha sido ampliamente estudiado en la literatura (por ejemplo Olander 1976; Dherbey et al. 2002; Vincent 2007; Julien 2008). Durante situaciones incidentales de

\footnotetext{
${ }^{1}$ Reactor de agua liviana a presión.
} 
operación ${ }^{2}$ que duran algunas horas, localmente en ciertas zonas del núcleo se produce un aumento de reactividad y temperatura en los poros presurizados por los gases producto de fisión, consecuentemente aumenta la presión dentro de los poros (Blair 2008) y la matríz de $\mathrm{UO}_{2}$ se dilata (véase Vincent 2009). Por otra parte, el efecto de la temperatura sobre la matríz tiende a contraer los poros. Dicha competencia produce una tasa de deformación tal que la matríz exhibe una respuesta viscoplástica; la fracción volumétrica de poros representa la variable microestructural clave que conecta ambos fenómenos.

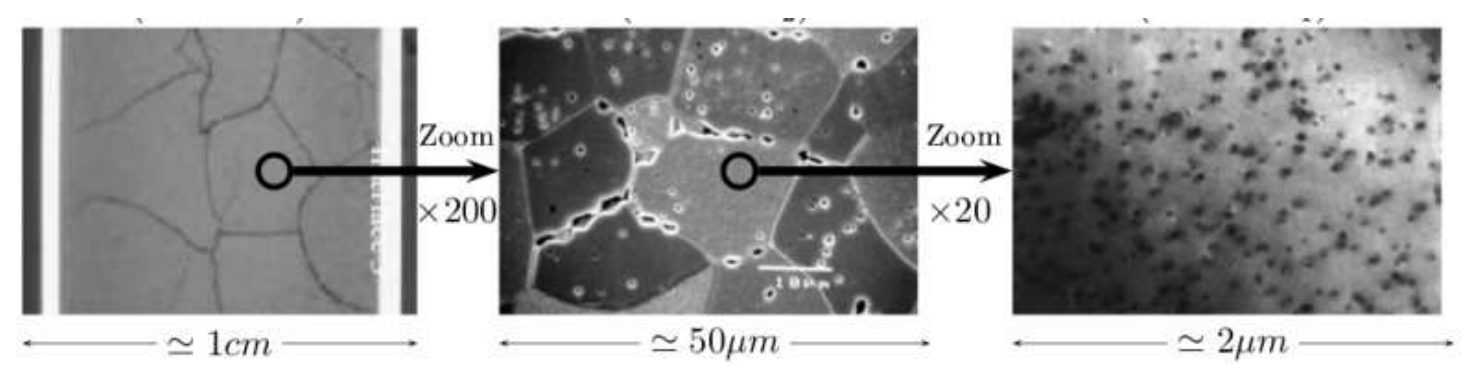

Figura 1.2: Pastilla de uranio enriquecido irradiada a altas dosis. Se observan las distintas poblaciones de poros a distintas escalas bién separadas. Dherbey et al. (2002).

Para el caso de las aplicaciones en sólidos metálicos cúbicos podemos clasificar los internos del reactor en aceros inoxidables austeníticos (cuya estructura cristalina es cúbica centrada en las caras, en la literatura inglesa FCC), aceros ferríticos (cúbicos centrado en el cuerpo -BCC-) y aleaciones base níquel (cúbicos centrado en las caras -FCC-). Los aceros inoxidables austeníticos cumplen una función estructural y sus propiedades mecánicas -durante la irradiación en el núcleo- van evolucionando producto de los cambios en la microestructura y la nucleación de porosidad presurizada (véase, por ejemplo, Maziasz 1987-1992-1993; Edwards 2003a-2009). Además exhiben endurecimiento y fragilización (véase, por ejemplo, Pokor et al. 2003 y Garnier 2007) y pérdida de tenacidad

\footnotetext{
${ }^{2}$ Transitorios de operación que implican una desestabilización parcial de la reactividad en el núcleo del reactor por un cambio de combustible por ejemplo.
} 
a la fractura (véase, por ejemplo, Torimaru et al. 2010, Jitsukawa et al. 1999, Rodchenkov et al. 2009). En la figura 1.3 se muestra el cambio en la hinchazón (conocido como gonflement en la literatura francesa) de un acero 316 irradiado a $600^{\circ} \mathrm{C}$ en el reactor Phénix, véase Dubuisson (2011), producto del aumento del dpa ${ }^{3}$. Esta hinchazón produce la deformación del componente deteriorando sus propiedades elastoplásticas y generando eventualmente un incremento de tensiones entre materiales y juntas soldadas. Los aceros ferríticos se utilizan fundamentalmente en recipientes de presión. Su evolución microestructural comprende nucleación de microporosidad y principalmente un cambio en la distribución y morfología de las partículas de segundas fases que producen endurecimiento y deterioran su tenacidad a la fractura (Kocik 2002; Nomoto 2014; Marta Serrano 2013; Soneda 2015). Este componente es vital porque constituye la superficie límite de presión del sistema primario de transporte de calor y posee límites de comportamiento mecánico bien establecidos por las normas nucleares. Por último, las aleaciones base níquel se utilizan por conservar su resiliencia bajo irradiación por tiempos más prolongados -respecto de otros materiales metálicos- por lo que es utilizada en aplicaciones de resortes y zapatas elásticas en los combustibles. En el caso de los reactores CANDU ${ }^{4}$, se utiliza como anillo separador ${ }^{5}$ y actualmente limitan la vida de los tubos de presión debido a que el níquel transmuta produciendo He que se aloja en la estructura de poros presurizados como la que se muestra en la figura 1.4 deteriorando la capacidad de restitución elástica de estos componentes hacia el fin de vida de la central (véase Zhang 2013). Por último las aleaciones de circonio (sólidos policristalinos hexagonales -HCP-) son utilizadas como componentes separadores de fluidos, elementos combustibles y límite de presión en el núcleo del reactor por su capacidad de baja sección eficaz de captura de neutrones. La irradiación neutrónica produce la difusión anisotrópica de vacancias hacia bordes de grano y de átomos autointersticiales hacia dislocaciones generando en

\footnotetext{
${ }^{3} \mathrm{El}$ dpa es una medida de daño producto de la exposición de los materiales a la irradiación. ${ }^{4}$ CANada Deuterium Uranium.

${ }^{5}$ Son anillos distribuidos a lo largo de los canales que separan el tubos de presión del tubo de calandria.
} 


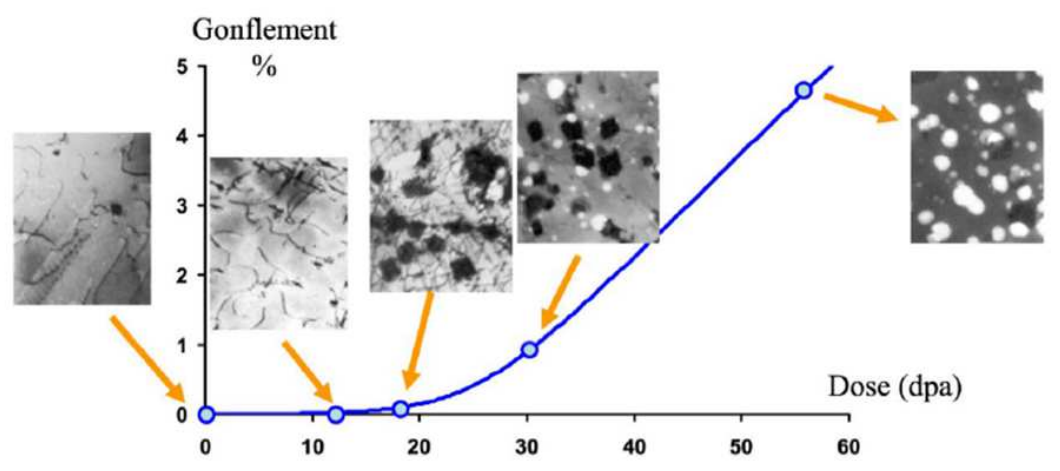

Figura 1.3: Efecto de la irradiación en la hinchazón (gonflement) de los aceros inoxidables austeníticos del tipo 316 irradiados a $600^{\circ} \mathrm{C}$ en el reactor Phénix, Dubuisson (2011).

promedio una deformación isocórica de la celda cristalina, fenómeno conocido como crecimiento por irradiación (Olander 1976; Holt 2004; Rogge 2006). Además compiten el creep térmico y el inducido por irradiación mediante diferentes mecanismos. La sinergia de estos fenómenos definen la deformación macroscópica la cual depende fuertemente de la anisotropía plástica del cristal simple y la textura cristalina.

El flujo plástico en sólidos policristalinos, dúctiles y porosos puede verse influenciado significativamente por la textura morfológica y cristalográfica del agregado (e.g., Caré \& Zaoui 1996, Hales \& Hafley 1998, Gray et al. 2000, Bache \& Evans 2001, Millet et al. 2008). El análisis teórico de este proceso requiere modelos micromecánicos que relacionen los estados de tensiones macroscópicos con las deformaciones plásticas microscópicas en los sistemas policristalinos porosos. Una simple aproximación utilizada, consiste en idealizar la respuesta mecánica de los granos individuales como elásticamente rígida y plásticamente sin endurecimiento, empleando técnicas de homogeneización para derivar la resistencia plástica macroscópica en términos de la resistencia plástica de los cristales simples, de la estadística, de la morfología y orientación y distribución de los granos y poros —véase la monografía de Kocks et al. (1998). Debido a su naturaleza microestructural inherentemente aleatoria, policristales semejantes con la misma estadística no 


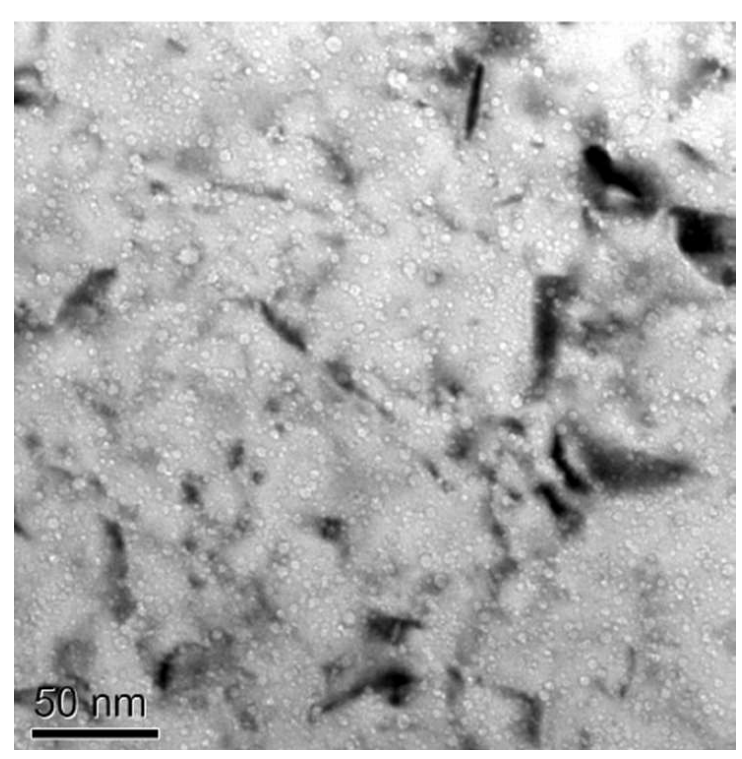

Figura 1.4: Micrografía de campo claro mostrando la formación de cavidades en un anillo espaciador. (Zhang 2013).

exhibirán una única respuesta macroscópica sino un rango de respuestas. Por lo tanto, se pueden desarrollar estimaciones que representen una respuesta representativa de un simple caso o derivar cotas para un rango entero de posibles respuestas. El interés de este trabajo es obtener cotas. Las cotas son también útiles por dos razones adicionales: proveen benchmarks para probar estimaciones y pueden ser utilizadas como estimaciones por sí mismas.

Existen varias técnicas de homogeneización disponibles para acotar la resistencia plástica de sólidos policristalinos. Su utilización, sin embargo, ha sido enfocada casi exclusivamente en sistemas materiales completamente densos, es decir, sin porosidad. La cota externa más simple es la de Taylor (1938) y la interna es la de Reuss (1929), las cuales dependen únicamente de estadísticas de primer orden. Estas cotas elementales han probado ser útiles en el contexto de sólidos policristalinos de gran simetría como cristales cúbicos FCC donde el contraste de heterogeneidad es bajo, pero al incrementarse la anisotropía cristalina sus predicciones divergen y se vuelven muy poco precisas. Haciendo uso de la 
idea de un medio lineal de comparación, se han derivado cotas incorporando estadísticas de orden superior, véase Dendievel et al. (1991) y deBotton \& Ponte Castañeda (1995), dicho medio es seleccionado óptimamente a través de principios variacionales diseñados específicamente. En particular, la técnica de deBotton \& Ponte Castañeda (1995) permite el uso de distintas técnicas de homogeneización lineal, como la de Hashin-Shtrikman o el Autoconsistente, para generar los correspondientes resultados para policristales no lineales. Se pueden encontrar en Willis (1994), Nebozhyn et al. (2000,2001), Liu et al. (2003), y Liu \& Ponte Castañeda (2004) la aplicación de dichos métodos lineales de comparación a varias clases de sólidos policristalinos. Dichos trabajos mostraron que las predicciones lineales de comparación pueden mejorar, a veces significativamente, a las predicciones elementales de Taylor y Reuss, y sirven para demostrar la inconsistencia de varias teorías de plasticidad policristalina basadas en esquemas de homogeneización ad-hoc. Luego, Idiart \& Ponte Castañeda (2007a,2007b) demostraron que la técnica lineal de comparación de deBotton \& Ponte Castañeda (1995) hace uso implícito de una relajación en el esquema variacional que debilita las cotas resultantes. Eliminando esa relajación se llega a cotas más estrictas a expensas de incrementar la complejidad computacional. El propósito de este trabajo es evaluar el comportamiento de las técnicas de homogeneización mencionadas anteriormente en el contexto de policristales porosos. Debido a su contraste en heterogeneidad infinitamente grande, son sistemas materiales particularmente difíciles de acotar ya que se espera que la aplicación de la mayoría de las técnicas produzca resultados con grandes divergencias. Ciertamente, es conocido que las cotas elementales se vuelven inútiles en presencia de fases porosas - la cota de Reuss se reduce al estado de tensiones idénticamente nulo, mientras que la de Taylor predice resistencia infinita bajo cargas puramente hidrostáticas - mientras que las cotas lineales de comparación brindan estimaciones significativas. Lebensohn et al. (2011) reportaron recientemente cotas lineales de comparación relajadas del tipo Autoconsistente para sistemas HCP porosos que exhiben una respuesta viscoplástica. Éstas sirven para discriminar entre dos teorías lineales de comparación de plasticidad policristalina -la secante y la secante generalizada, pero brindan predicciones lejanas a las soluciones exactas obtenidas por simulaciones 


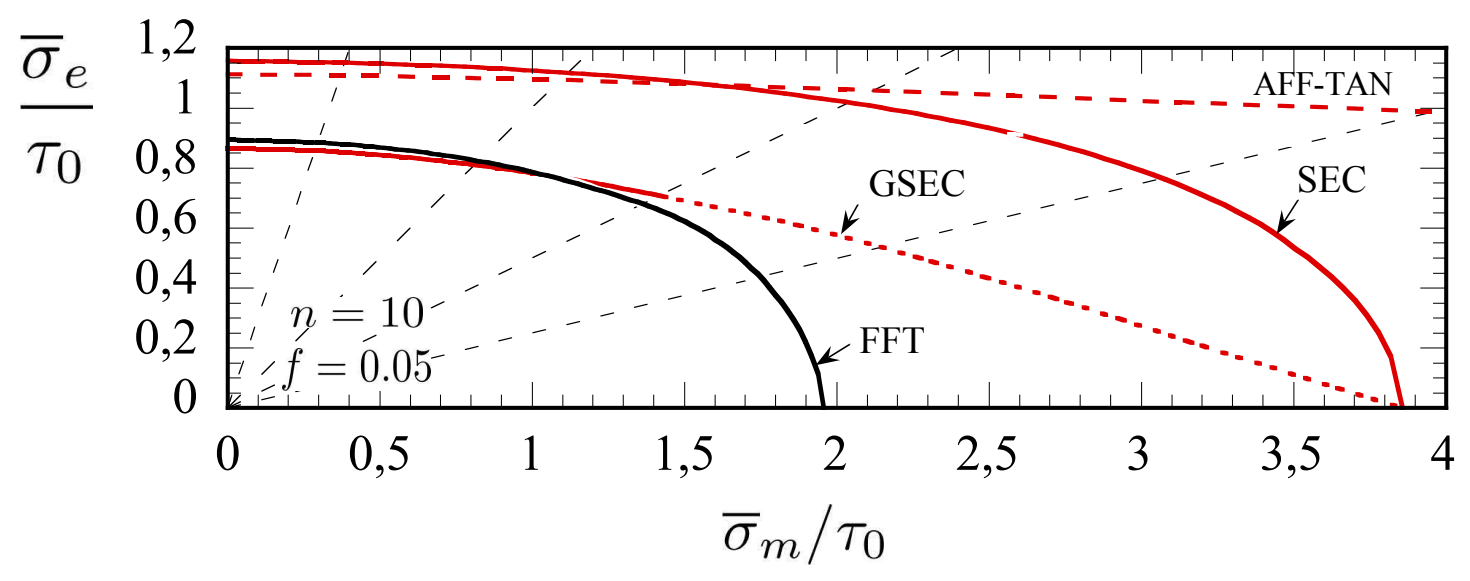

Figura 1.5: Estimaciones secante (SEC) y secante generalizada (GSEC) para las superficies de fluencia de sólidos policristalinos hexagonales con moderado nivel de porosidad $(f=0,05)$ y exponente viscoso $n=10$. Además se presentan simulaciones de transformada rápida de Fourier (FFT) y la estimación afín tangencial (AFFTAN) Autoconsistente para la comparación de los resultados. Las líneas a tramos representan las direcciones de triaxilidad constante $\bar{X}=1 / 3,1,2,4$. Cargas axisimétricas $\bar{\theta}=0$ Lebenshon (2011).

basadas en la transformada rápida de Fourier (FFT por sus siglas en inglés). Esto puede observarse en la figura 1.5 donde se representan las superficies de fluencia efectiva ${ }^{6}$ de policristales con cinco sistemas de deslizamiento linealmente independientes, sin textura, con un moderado nivel de porosidad $(f=0,05)$ y un exponente viscoplástico de $\mathrm{n}=10$. La estimación Autoconsistente tangente obtenida mediante el método afín (AFF-TAN) produce una superficie de fluencia no convexa y extremadamente rígida para cargas con alta triaxialidad. Las estimaciones obtenidas por el método de la secante (SEC) y la secante generalizada (GSEC) coinciden en la resistencia hidrostática mientras que únicamente la GSEC brinda predicciones cercanas a los resultados exactos para bajas triaxialidades $\bar{X}<2$. En el artículo de Idiart (2012) se discutieron las cotas obtenidas por metodologías de comparación lineal derivadas originalmente en Idiart \& Ponte Castañeda (2007a, b)

\footnotetext{
${ }^{6}$ Estrictamente no son superficies de fluencia, se denominan gauge surfaces en inglés. Se representa en función de los invariantes del tensor de tensiones: resistencia hidrostática $\bar{\sigma}_{m} \mathrm{y}$ tensión equivalente $\bar{\sigma}_{e}$ normalizando con la resistencia a la fluencia $\tau_{0}$ a nivel cristalino y se definirán en el Capítulo 4.
} 
- en un contexto más general de viscoplasticidad que incluye la respuesta rígida perfectamente plástica como caso extremo - con otras cotas disponibles en la literatura para el caso de policristales sin porosidad y varios grados de anisotropía cristalina. Se encontraron mejoras modestas para el caso de cristales de baja simetría pero mejoras de hasta $15 \%$ en materiales con alto grado de anisotropía (HCP). Es por ello que se espera hallar en este trabajo mejoras más significativas ya que se consideran policristales porosos que representan el caso extremo de contraste entre fases. En consecuencia se espera en el caso de sistemas policristalinos porosos - donde el contraste es extremoque la mejora sea aún mayor que en caso de Idiart (2012) produciendo un aumento en el rango de triaxialidades para el cual las cotas brindan predicciones precisas. Es decir, se espera un corrimiento en la curva SEC y por consiguiente la GSEC ampliaría el rango de triaxialidades $(\bar{X}>2)$ para el cual las estimaciones coinciden con las soluciones exactas; aún cuando este método está siendo utilizado en materiales con un exponente de viscoplasticidad infinito ${ }^{7}$.

En este trabajo, se reportan cotas lineales de comparación del tipo Hashin-Shtrikman y Autoconsistente ${ }^{8}$ para sistemas cúbicos y HCP de policristales porosos con textura isótropa y varios grados de anisotropía cristalina, sujetos a condiciones de carga axisimétrica. Debido a que el análisis trata de la misma forma a fases porosas y cristalinas, los resultados de este trabajo son relevantes para sistemas materiales con porosidad intergranular; la porosidad intragranular debe ser tratada en forma diferente - por ejemplo, Han et al. (2013). Se pone especial atención al juego entre la cristalinidad, porosidad y el impacto en las cotas de las relajaciones variacionales.

En el Capítulo 2 se describe el modelo policristalino con cavidades y la superficie de fluencia macroscópica derivada por Suquet (1983) y Bouchitté \& Suquet (1991), se

\footnotetext{
${ }^{7} \mathrm{~A}$ modo comparativo, el caso de viscoplasticidad con un exponente $\mathrm{n}=10$ es en general una aproximación al caso de plasticidad perfecta donde el $\mathrm{n}$ tiende a infinito.

${ }^{8}$ Estrictamente, el uso de la metodología de Hashin-Shtrikman provee cotas para una clase entera de policristales con estadísticas prescriptas de orden uno y dos, mientras que el uso de la metodología Autoconsistente provee cotas para una subclase de policristales que da cuenta del esquema Autoconsistente.
} 
presentan los sistemas materiales específicos a considerarse y se describe el tratamiento de la presión interna de las cavidades. En el Capítulo 3 se derivan las cotas utilizando métodos basados en medios lineales de comparación, las cotas más estrictas resultan las presentadas en Idiart (2007a-b) y se recuperan resultados de cotas relajadas provenientes de relajaciones implícitas en los esquemas variacionales como es el caso de las cotas de deBotton \& Ponte Castañeda (1995). Utilizando principios variacionales de HashinShtrikman, se lineariza la respuesta del policristal no lineal obteniendo el medio lineal de comparación. Luego se aplican técnicas de homogeneización lineal con los métodos de Hashin-Shtrikman y Autoconsistente -en sus versiones relajadas y no relajadas ${ }^{9}-$ obteniendo de este modo el potencial efectivo (homogeneizado) del policristal lineal. Se presenta por último el cálculo de la resistencia hidrostática para el caso de materiales isótropos; es decir, las cotas de resistencia hidrostática estrictas de Hashin-Shtrikman y Autoconsistente y sus versiones relajadas.

En el Capítulo 4 se presenta la aplicación de las cotas a los distintos sistemas materiales evaluando el efecto de la cristalinidad. Se obtienen superficies de fluencia -a una porosidad de $f=0,05$ - para el caso de materiales perfectamente plásticos y elásticamente rígidos con textura isótropa; éstas son superficies suaves y cerradas presentando mejoras de hasta un $40 \%$ respecto de las versiones relajadas. Se evalúa el efecto de la porosidad en la resistencia hidrostática y al corte para todas las cotas consideradas obteniendo leyes de escalamiento generales que recuperan los resultados inferidos por Nebozhyn et al. (2001) para la resistencia al corte en policristales sin porosidad. Además, se evalúa el efecto del contraste entre fases de un mismo sistema cristalino variando la resistencia al deslizamiento $^{10}$ y en consecuencia los sistemas de deslizamiento disponibles. Por último se observa el exponente de la porosidad respecto de la resistencia hidrostática para el caso diluído, la capacidad de captar dicho exponente es una característica destacable de los métodos de medio de comparación lineal utilizados. En el quinto capítulo se presentan las conclusiones de la tesis específicas a los resultados.

\footnotetext{
${ }^{9}$ Las no relajadas corresponden a las derivadas por Idiart (2007a-b).

${ }^{10}$ Es la denominada tensión crítica resuelta por plano cristalino.
} 
En el Apéndice A se presenta la notación utilizada para la representación de tensores; los resultados analíticos de las cotas de resistencia hidrostática para el caso diluído; los tensores vértice que representan el estado de tensiones en las esquinas del polihedro de superficie de fluencia local; detalles del tensor de flexibilidad efectivo y del procedimiento de optimización para el cálculo de cotas. En el Apéndice B se muestran los cálculos de la resistencia hidrostática para sólidos con sistemas deficientes, se colocaron en este apartado por similitud en el procedimiento de cálculo respecto de policristales sin sistemas deficientes. 



\section{Capítulo 2}

\section{Modelo de policristales porosos presurizados}

\subsection{Modelo del sólido poroso policristalino}

En este trabajo los policristales porosos se idealizan como una distribución aleatoria de agregados de cavidades y cristales simples perfectamente cohesionados (granos). Se asume que individualmente los granos y las cavidades son de similar tamaño, mucho más pequeños que la escala de variación de las cargas aplicadas y del tamaño de la muestra en consideración. Por último, se asume que los agregados son estadísticamente uniformes y ergódicos.

La respuesta plástica se estudia convenientemente adoptando una descripción Euleriana del movimiento; por lo tanto el presente análisis se refiere a la configuración actual del agregado en un estado de deformación general. La orientación de los granos, en la configuración actual, está definida como un conjunto de $N$ valores discretos caracterizados por tensores de rotación $\mathbf{Q}^{(r)}(r=1, \ldots, N)$. Todos los granos con una dada orientación $\mathbf{Q}^{(r)}$ ocupan un dominio no-conexo $\Omega^{(r)}$ y están referenciadas colectivamente como 'fase' $r$. De manera análoga, todas las cavidades ocupan un dominio no-conexo $\Omega^{(0)}$ y están referenciadas colectivamente como 'fase' 0. El dominio ocupado por el policristal 
es $\Omega=\cup_{r=0}^{N} \Omega^{(r)}$. Los dominios $\Omega^{(r)}$ son descriptos por el conjunto de funciones características $\chi^{(r)}(\mathbf{x})$, las cuales valen 1 si la posición $\mathbf{x}$ pertenece al dominio $\Omega^{(r)}$ y 0 en caso contrario.

Se asume que los granos $(r=1, \ldots, N)$ se deforman de manera individual a lo largo de $K$ sistemas de deslizamiento presentando una respuesta elásticamente rígida y perfectamente plástica. Las cavidades, en cambio, no pueden sustentar cargas. Por simplicidad, los efectos de la elasticidad de los granos y efectos de maclado se desprecian en la presente formulación. De acuerdo a la teoría estándar de plasticidad cristalina, los dominios de resistencia plástica se caracterizan en términos de conjuntos convexos

$$
P^{(r)}=\left\{\boldsymbol{\sigma}:\left|\boldsymbol{\sigma} \cdot \boldsymbol{\mu}_{(k)}^{(r)}\right| \leq \tau_{0}^{(k)}, k=1, \ldots, K\right\}
$$

donde $\tau_{0}^{(k)}>0$ es la resistencia a la fluencia del k-ésimo sistema de deslizamiento en un cristal de referencia y

$$
\boldsymbol{\mu}_{(k)}^{(r)}=\frac{1}{2}\left(\mathbf{n}_{(k)}^{(r)} \otimes \mathbf{m}_{(k)}^{(r)}+\mathbf{m}_{(k)}^{(r)} \otimes \mathbf{n}_{(k)}^{(r)}\right)
$$

son los tensores de Schmid de segundo orden con $\mathbf{n}_{(k)}^{(r)}$ y $\mathbf{m}_{(k)}^{(r)}$ representando el vector unidad normal al plano de deslizamiento y a lo largo de la dirección del $k$-ésimo sistema, respectivamente, para un determinado cristal con orientación $\mathbf{Q}^{(r)}$. Los tensores de Schmid de una dada fase $r$ están relacionados con el correspondiente tensor $\boldsymbol{\mu}_{(k)}$ para el cristal de referencia a través de

$$
\boldsymbol{\mu}_{(k)}^{(r)}=\mathbf{Q}^{(r)^{T}} \boldsymbol{\mu}_{(k)} \mathbf{Q}^{(r)}
$$

Nótese que los tensores de Schmid poseen traza nula y por lo tanto los dominios de resistencia plástica (2.1) son insensibles a las tensiones hidrostáticas. El conjunto $P$ es un polihedro convexo formado por el conjunto de planos (o caras) cuyas ecuaciones están dadas por las desigualdades $(2.1)$. El conjunto de vértices de $P^{(n)}$ se denota $\hat{P}^{(n)}$. El borde $\partial P^{(r)}$ del conjunto $P^{(r)}$ representa la superficie de fluencia de la fase $r$. El flujo plástico de los granos es gobernado por la llamada regla de la normal. La fase porosa $(r=0)$, por otra parte, no soporta tensiones y está sometida a una presión interna $p_{0}$. 
Caracterizaremos esta fase como una familia adicional de 'granos' con

$$
P^{(0)}=\left\{-p_{0} \mathbf{I}\right\}
$$

Nótese que se está asumiendo que los poros tienen todos la misma presión.

Los promedios volumétricos sobre los agregados en $\Omega$ y sobre cada fase $\Omega^{(r)}$ se denotarán como $\langle\cdot\rangle$ y $\langle\cdot\rangle^{(r)}$, respectivamente. Debido a que los policristales en estudio poseen una distribución al azar de microestructura, las funciones $\chi^{(r)}$ son variables al azar que deben ser caracterizadas en términos de promedios estadísticos (Willis 1977). El promedio de $\chi^{(r)}(\mathbf{x})$ representa la probabilidad total de primer orden $p^{(r)}(\mathbf{x})$ de hallar la fase $r$ en la posición $\mathbf{x}$; el promedio del producto $\chi^{(r)}(\mathbf{x}) \chi^{(s)}\left(\mathbf{x}^{\prime}\right)$ representa las probabilidades de segundo orden $p^{(r s)}\left(\mathbf{x}, \mathbf{x}^{\prime}\right)$ de hallar simultáneamente la fase $r$ en la posción $\mathbf{x}$ y la fase $s$ en la posición $\mathbf{x}^{\prime}$. Similarmente se pueden definir probabilidades de orden superior. Debido a la uniformidad estadística y la ergodicidad asumidas, las probabilidades de primer orden $p^{(r)}(\mathbf{x})$ pueden ser identificadas con las fracciones de volumen (o concentraciones) $c^{(r)}=\left\langle\chi^{(r)}(\mathbf{x})\right\rangle$ de cada fase $r$, la probabilidad de segundo orden $p^{(r s)}\left(\mathbf{x}, \mathbf{x}^{\prime}\right)$ puede ser identificada con el volumen promedio $\left\langle\chi^{(r)}(\mathbf{x}) \chi^{(s)}\left(\mathbf{x}^{\prime}\right)\right\rangle$, y así sucesivamente. Nótese que $\sum_{r=0}^{N} c^{(r)}=1$. Por otro lado, al describir policristales porosos resulta conveniente utilizar el conjunto de concentraciones (Lebensohn et al. 2011):

$$
f=c^{(0)}, \quad \text { y } \quad c_{g}^{(r)}=\frac{c^{(r)}}{1-f} \quad \text { para } r=1, \ldots, N .
$$

La variable microestructural $f$ representa la fracción en volumen de las cavidades, o porosidad, en el policristal con cavidades, mientras que las concentraciones de granos reescaladas $c_{g}^{(r)}$ representan la fracción en volumen de los granos con una dada orientación $\mathbf{Q}^{(r)}$ dentro de la matríz policristalina, las concentraciones son tales que $\sum_{r=1}^{N} c_{g}^{(r)}=1$. Por lo tanto, el conjunto de fracciones de volumen $c_{g}^{(r)}$ caracterizan la textura cristalográfica del agregado alrededor de los poros, mientras que las funciones de correlaciones de orden superior caracterizan la textura morfológica del agregado y el aspecto y distribución de cavidades.

La resistencia plástica macroscópica del agregado policristalino corresponde al conjunto de estados de tensiones que pueden producir flujo plástico. Homogeneizando las 
ecuaciones de campo apropiadas, Suquet (1983) y Bouchitté \& Suquet (1991) mostraron que la resistencia plástica macroscópica puede ser caracterizada por un dominio efectivo de fluencia definido como

$$
\widetilde{P}=\left\{\overline{\boldsymbol{\sigma}}: \exists \boldsymbol{\sigma}(\mathbf{x}) \in \mathcal{S}(\overline{\boldsymbol{\sigma}}) \text { and } \boldsymbol{\sigma}(\mathbf{x}) \in P^{(r)} \text { in } \Omega^{(r)}, r=0, \ldots, N\right\}
$$

donde $\overline{\boldsymbol{\sigma}}$ representa el estado de tensiones macroscópico que puede producir flujo plástico, $\boldsymbol{\sigma}(\mathbf{x})$ son los correspondientes campos de tensiones microscópicos, y

$$
\mathcal{S}(\overline{\boldsymbol{\sigma}})=\{\boldsymbol{\sigma}(\mathbf{x}): \operatorname{div} \boldsymbol{\sigma}(\mathbf{x})=\mathbf{0} \text { in } \Omega,\langle\boldsymbol{\sigma}(\mathbf{x})\rangle=\overline{\boldsymbol{\sigma}}\}
$$

es el conjunto de campos de tensiones estáticamente admisibles con promedio volumétrico $\overline{\boldsymbol{\sigma}}$. En consecuencia, el dominio plástico efectivo depende de la textura cristalográfica del policristal a través del conjunto de orientaciones $\mathbf{Q}^{(r)}$ y concentraciones $c_{g}^{(r)}$, de la textura morfológica a través del promedio de las funciones caracteríticas $\chi^{(r)}(\mathbf{x})$ de los dominios $\Omega^{(r)}$, y de la porosidad a través de $f$. Nótese que la convexidad de los conjuntos $P^{(r)}$ implica convexidad de $\widetilde{P}$. El borde $\partial \widetilde{P}$ del conjunto $\widetilde{P}$ representa la superficie de fluencia efectiva del sólido policristalino poroso, superficie que se busca acotar.

\subsection{Algunos sistemas materiales}

Los sistemas materiales considerados en este trabajo son:

- sólidos cúbicos centrado en la cara (de ahora en adelante los denominamos FCC por la literatura inglesa) que se deforman por deslizamiento plástico en un conjunto de cuatro planos de deslizamiento del tipo $\{111\}$ a lo largo de tres direcciones de deslizamiento (por plano) del tipo $\langle 110\rangle$, lo cual constituye un conjunto de doce sistemas de deslizamiento $(K=12)$. De ellos, cinco son linealmente independientes, permitiendo acomodar una deformación plástica arbitraria de los granos. Este conjunto de sistemas de deslizamiento define una superficie de fluencia con 56 vértices (véase el Anexo A). 
- sólidos cúbicos centrado en el cuerpo (de ahora en adelante los denominamos BCC por la literatura inglesa) BCC que deforman por deslizamiento plástico a lo largo de las direcciones $\langle 111\rangle$ en los planos $\{110\}$ y $\{112\}$ —el trepado a lo largo de los planos $\{123\}$ no es considerado-, lo cual constituye un conjunto de 24 sistemas de deslizamiento $(K=24)$. De ellos, cinco son linealmente independientes, permitiendo acomodar una deformación plástica arbitraria de los granos. Este conjunto de sistemas de deslizamiento define una superficie de fluencia con 132 vértices.

- sólidos iónicos (de ahora en adelante los denominamos IONIC por la literatura inglesa) que deforman por deslizamiento plástico en tres diferentes familias de planos de deslizamiento: $\{110\}\langle 110\rangle,\{100\}\langle 110\rangle,\{111\}\langle 110\rangle$. Nos referiremos a las familias como $A, B$ y $C$. La familia $A$ consiste en seis sistemas, entre los cuales dos son linealmente independientes y pueden acomodar únicamente las componentes normales de la tasa de deformación - con respecto a los ejes cúbicos del cristal. La familia $B$ consiste en seis sistemas, entre los cuales tres son linealmente independientes y pueden únicamente acomodar tasas de deformaciones de corte - con respecto a los ejes cúbicos del cristal. Debido a la ortogonalidad de los sistemas $A$ y $B$, ambas familias juntas proveen cinco sistemas de deslizamiento independientes por lo que pueden acomodar una deformación isocórica general. La familia $C$, consiste de los mismos 12 sistemas de deslizamiento de un cristal FCC. Por lo tanto, las tres familias juntas proveen 24 sistemas de deslizamiento $(K=24)$. Este conjunto de sistemas de deslizamiento define una superficie de fluencia con 312 vértices.

- sólidos hexagonales compactos (de ahora en adelante los denominamos HCP por la literatura inglesa) que deforman por deslizamiento plástico en tres diferentes sistemas de deslizamiento: tres sistemas basales $\{0001\}\langle 11 \overline{2} 0\rangle$, tres sistemas prismáticos $\{10 \overline{1} 0\}\langle 11 \overline{2} 0\rangle$, y doce sistemas piramidales de primer orden- $\langle c+a\rangle\{10 \overline{11}\}\langle 11 \overline{2} 3\rangle$. Los denotaremos como las familias $A, B$ y $C$, que poseen una tensión de fluencia $\tau_{A}, \tau_{B}$ y $\tau_{C}$, respectivamente. Nótese que los tres sistemas basales más los tres sistemas prismáticos brindan solamente cuatro $(J=4)$ sistemas linealmente 
independientes, no permitiendo la deformación a lo largo de los ejes del cristal hexagonal. Por otra parte, los doce sistemas piramidales contienen un conjunto de cinco sistemas independientes, permitiendo acomodar una deformación plástica arbitraria de los granos. Las tres familias juntas proveen un conjunto de 18 sistemas de deslizamiento $(K=18)$. Este conjunto de sistemas de deslizamiento define una superficie de fluencia con 306 vértices.

\subsection{Efecto de la presión interna en la superficie de fluencia del policristal}

La resistencia plástica efectiva del policristal con cavidades presurizadas se puede obtener a partir de la resistencia plástica del policristal con cavidades vacías realizando un cambio de variables adecuado. En $(2.4)$ se vio que $\widetilde{P}$ es de la forma

$$
\widetilde{P}\left(p_{0}\right)=\left\{\overline{\boldsymbol{\sigma}}: \exists \boldsymbol{\sigma}(\mathbf{x}) \in \mathcal{S}(\overline{\boldsymbol{\sigma}}) \text { and } \boldsymbol{\sigma}(\mathbf{x}) \in P^{(r)} \text { in } \Omega^{(r)}, r=0, \ldots, N\right\} .
$$

Sea $\boldsymbol{\sigma}^{\prime}(\mathbf{x}) \doteq \boldsymbol{\sigma}(\mathbf{x})+p_{0} \mathbf{I}$. Se puede demostrar fácilmente que $\boldsymbol{\sigma}^{\prime}(\mathbf{x})$ es tal que:

$$
\operatorname{div} \boldsymbol{\sigma}^{\prime}(\mathbf{x})=\mathbf{0} \quad \text { y } \quad \overline{\boldsymbol{\sigma}}^{\prime}=\overline{\boldsymbol{\sigma}}+p_{0} \mathbf{I},
$$

y por lo tanto que

$$
\boldsymbol{\sigma}^{\prime}(\mathbf{x}) \in \mathcal{S}\left(\overline{\boldsymbol{\sigma}}+p_{0} \mathbf{I}\right)
$$

Vale decir que $\boldsymbol{\sigma}^{\prime}(\mathbf{x})$ cumple también con las condiciones de campos estáticamente admisibles con el promedio volumétrico $\bar{\sigma}$ a menos de una constante que es el campo hidrostático de presiones. Además se cumple que las proyecciones de ambos campos de tensiones sobre los planos de deslizamiento coinciden ya que difieren únicamente de una componente hidrostática:

$$
\boldsymbol{\sigma}^{\prime}(\mathbf{x}) \cdot \boldsymbol{\mu}_{(k)}^{(r)}=\boldsymbol{\sigma}(\mathbf{x}) \cdot \boldsymbol{\mu}_{(k)}^{(r)}
$$




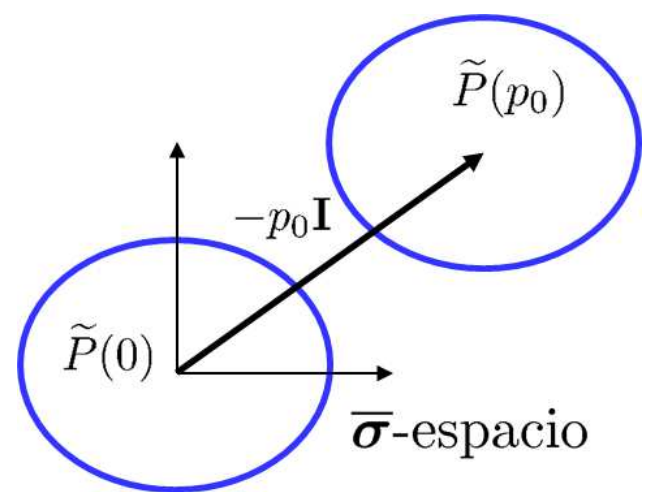

Figura 2.1: Efecto de la porosidad en la superficie de fluencia: en el espacio de tensiones, la superficie de fluencia se traslada una magnitud equivalente a $p_{0} \mathbf{I}$ por efecto de la presurización de las cavidades.

Vale decir que $\boldsymbol{\sigma}^{\prime}(\mathbf{x}) \in P^{(r)}$ para todas las fases cristalinas $r=1, \ldots, N$ y $\boldsymbol{\sigma}^{\prime}(\mathbf{x})=\mathbf{0}$ para las cavidades. Por lo tanto:

$$
\widetilde{P}\left(p_{0}\right)=\left\{\overline{\boldsymbol{\sigma}}: \overline{\boldsymbol{\sigma}}=\overline{\boldsymbol{\sigma}}^{\prime}-p_{0} \mathbf{I}, \overline{\boldsymbol{\sigma}}^{\prime} \in \widetilde{P}(0)\right\} .
$$

Es decir, la superficie de resistencia plástica del problema presurizado corresponde a una traslación de la superficie de fluencia del problema sin presurizar como se representa esquemáticamente en la figura 2.1. En consecuencia, de aquí en más dejaremos de lado la presión de las cavidades. Las superficies a obtener serán válidas para el problema presurizado trasladándolas una cantidad proporcional a la presión interna de los poros. 



\section{Capítulo 3}

\section{Cotas para superficies de fluencia de policristales porosos}

\subsection{Métodos basados en medios lineales de com- paración}

Las cotas externas para la superficie de fluencia (2.4) se obtienen en este trabajo utilizando la técnica del policristal lineal de comparación presentada en Idiart \& Ponte Castañeda (2007a,b). Este trabajo continúa el presentado en Idiart (2012) adaptado al caso de policristales porosos. La idea principal detrás de la técnica es introducir un policristal lineal de comparación con los mismos dominios microestructurales $\chi^{(r)}$ que posee el policristal original (no lineal) pero con una relación local lineal de la tensióntasa de deformación caracterizada por un tensor de flexibilidad de cuarto orden positivo semidefinido ${ }^{1} \mathrm{y}$ simétrico.

El potencial de tensiones $u^{(r)}(\boldsymbol{\sigma})$ en cada dominio elástico correspondiente a las $n$ fases del agregado se define como:

${ }^{1}$ La característica de un tensor de flexibilidad $\mathbb{S}^{(r)}$ de cuarto orden de ser positivo semidefinido $\mathbb{S}$ se indica por la desigualdad $\mathbb{S} \geq 0$. 


$$
u^{(r)}(\boldsymbol{\sigma})= \begin{cases}0 & \boldsymbol{\sigma} \in P^{(r)} \\ +\infty & \text { c.c. }\end{cases}
$$

Mientras que definimos el potencial $\widetilde{u}(\overline{\boldsymbol{\sigma}})$ efectivo del policristal no lineal como:

$$
\widetilde{u}(\overline{\boldsymbol{\sigma}})= \begin{cases}0 & \overline{\boldsymbol{\sigma}} \in \widetilde{P} \\ +\infty & \text { c.c. }\end{cases}
$$

El potencial $\widetilde{u}(\overline{\boldsymbol{\sigma}})$ es solución del problema de optimización (véase Bouchitté, G. \& Suquet, P. 1991)

$$
\widetilde{u}(\overline{\boldsymbol{\sigma}})=\min _{\boldsymbol{\sigma} \in \mathcal{S}^{*}(\overline{\boldsymbol{\sigma}})}\langle u(\mathbf{x}, \boldsymbol{\sigma})\rangle, \quad \text { donde } \quad u(\mathbf{x}, \boldsymbol{\sigma})=\sum_{r=1}^{N} \chi^{(r)}(\mathbf{x}) u^{(r)}(\boldsymbol{\sigma}) .
$$

Elegimos el potencial de tensiones local del medio lineal de comparación $u_{0}(\mathbf{x}, \boldsymbol{\sigma})$ homogéneo de segundo grado en el campo local $\boldsymbol{\sigma}$

$$
u_{0}(\mathbf{x}, \boldsymbol{\sigma})=\frac{1}{2} \boldsymbol{\sigma} \cdot \mathbb{S}_{0}(\mathbf{x}) \boldsymbol{\sigma}
$$

Acotando el potencial $\widetilde{u}$ puede acotarse el dominio $\widetilde{P}$. Para ello se define una función:

$$
v\left(\mathbb{S}_{0}\right) \doteq \sup _{\boldsymbol{\sigma} \in \mathcal{S}^{*}(\overline{\boldsymbol{\sigma}})}\left[u_{0}(\mathbf{x}, \boldsymbol{\sigma})-u(\mathbf{x}, \boldsymbol{\sigma})\right] \geq u_{0}(\mathbf{x}, \boldsymbol{\sigma})-u(\mathbf{x}, \boldsymbol{\sigma}),
$$

que representan una medida de la no linearidad de la relación plástica local de tensión tasa de deformación. Se observa de la ecuación (3.5) que se cumple la siguiente desigualdad:

$$
u(\mathbf{x}, \boldsymbol{\sigma}) \geq u_{0}(\mathbf{x}, \boldsymbol{\sigma})-v\left(\mathbb{S}_{0}\right), \quad \forall \boldsymbol{\sigma}, \mathbb{S}_{0}>0 .
$$

Reemplazando la desigualdad (3.6) en (3.3) se obtiene:

$$
\widetilde{u}(\overline{\boldsymbol{\sigma}}) \geq \min _{\boldsymbol{\sigma} \in \mathcal{S}^{*}(\overline{\boldsymbol{\sigma}})}\left\langle u_{0}(\mathbf{x}, \boldsymbol{\sigma})-v\left(\mathbb{S}_{0}\right)\right\rangle=\min _{\boldsymbol{\sigma} \in \mathcal{S}^{*}(\overline{\boldsymbol{\sigma}})}\left\langle u_{0}(\mathbf{x}, \boldsymbol{\sigma})\right\rangle-\left\langle v\left(\mathbb{S}_{0}\right)\right\rangle .
$$

Por lo tanto,

$$
\widetilde{u}(\overline{\boldsymbol{\sigma}}) \geq \widetilde{u}_{0}\left(\overline{\boldsymbol{\sigma}}, \mathbb{S}_{0}\right)-\left\langle v\left(\mathbb{S}_{0}\right)\right\rangle, \quad \forall \mathbb{S}_{0}>0
$$


La ecuación (3.8) se cumple en particular para $\mathbb{S}_{0}$ de la forma:

$$
\mathbb{S}_{0}(\mathbf{x})=\sum_{r=1}^{N} \mathbb{S}_{0}^{(r)} \chi^{(r)}(\mathbf{x}) .
$$

Para la opción (3.9) de $\mathbb{S}_{0}(\mathbf{x})$ el potencial efectivo del policristal no lineal cumple:

$$
\widetilde{u}(\overline{\boldsymbol{\sigma}}) \geq \sup _{\mathbb{S}_{0} \geq 0}\left[\widetilde{u}_{0}\left(\overline{\boldsymbol{\sigma}}, \mathbb{S}_{0}\right)-\left\langle v\left(\mathbb{S}_{0}\right)\right\rangle\right]=\widetilde{u}_{-} .
$$

El potencial efectivo de tensiones $\widetilde{u}$ del policristal no lineal queda acotado por debajo por el potencial $\widetilde{u}_{-}$que es la solución del problema de optimización del miembro derecho de la desigualdad (3.10). Esto implica una cota exterior en el conjunto de tensiones que definen el dominio elástico macroscópico efectivo del policristal no lineal (Idiart 2007a), es decir:

$$
\widetilde{P} \subset \widetilde{P}_{+}=\left\{\overline{\boldsymbol{\sigma}}: \widetilde{u}_{0}\left(\overline{\boldsymbol{\sigma}} ; \mathbb{S}^{(s)}\right) \leq v\left(\mathbb{S}^{(s)}\right), \forall \mathbb{S}^{(r)} \geq 0(r=1, \ldots, N)\right\}
$$

Para el caso de policristales porosos los miembros de la ecuación (3.11) son

$$
\begin{aligned}
\widetilde{u}_{0}\left(\overline{\boldsymbol{\sigma}} ; \mathbb{S}^{(s)}\right) & =(1-f) \min _{\boldsymbol{\sigma} \in \mathcal{S}^{*}(\overline{\boldsymbol{\sigma}})} \sum_{r=1}^{N} c_{g}^{(r)}\left\langle\frac{1}{2} \boldsymbol{\sigma} \cdot \mathbb{S}^{(r)} \boldsymbol{\sigma}\right\rangle^{(r)} \\
v\left(\mathbb{S}^{(s)}\right) & =(1-f) \sum_{r=1}^{N} c_{g}^{(r)} v^{(r)}\left(\mathbb{S}^{(r)}\right)
\end{aligned}
$$

con

$$
v^{(r)}\left(\mathbb{S}^{(r)}\right)=\sup _{\boldsymbol{\sigma} \in P^{(r)}} \frac{1}{2} \boldsymbol{\sigma} \cdot \mathbb{S}^{(r)} \boldsymbol{\sigma}=\operatorname{máx}_{\boldsymbol{\sigma} \in \hat{P}^{(r)}}\left\{\frac{1}{2} \boldsymbol{\sigma} \cdot \mathbb{S}^{(r)} \boldsymbol{\sigma}\right\} .
$$

El conjunto $\mathcal{S}^{*} \subset \mathcal{S}$ denota el subconjunto de campos de tensiones estáticamente admisibles con tracción cero en las superficies $\partial \Omega^{(0)}$ de los poros. La ventaja de escribir las funciones $v^{(r)}$ como en la primera igualdad de la ecuación (3.14) es que su cálculo se reduce al conocido problema de hallar el máximo de una función convexa relativo a un conjunto convexo. De hecho, puede demostrarse -véase el corolario 32.3.2 en Rockafellar (1970) - que el máximo en la primera igualdad de la 3.14 se alcanzará en uno o más de los puntos extremos del conjunto $P^{(r)}$, lo que significa que el valor óptimo de $\boldsymbol{\sigma}^{(r)}$ está necesariamente en la superficie de fluencia de la r-ésima fase. En el caso de las fases cristalinas el número de puntos extremos de $P^{(r)}$ es finito y la función $v^{(r)}$ puede escribirse 
como en la última igualdad de la (3.14). Nótese que las maximizaciones en las funciones $v^{(r)}$ reducen la evaluación de la función objetivo sobre un conjunto finito de puntos. El borde de $\widetilde{P}_{+}$representa la superficie en el espacio de tensiones macroscópicos que acota desde el exterior la superficie de fluencia efectiva del sólido policristalino poroso; el mismo puede escribirse como

$$
\partial \widetilde{P}_{+}=\left\{\overline{\boldsymbol{\sigma}}: \overline{\boldsymbol{\sigma}}=\Lambda \overline{\boldsymbol{\Sigma}} \text { with }\|\overline{\boldsymbol{\Sigma}}\|=1 \text { and } \Lambda=\inf _{\mathbb{S}^{(r)} \geq 0}\left(\frac{\widetilde{u}_{0}\left(\overline{\boldsymbol{\Sigma}} ; \mathbb{S}^{(s)}\right)}{v\left(\mathbb{S}^{(s)}\right)}\right)^{-1 / 2}\right\},
$$

donde $\|\cdot\|$ representa la norma Euclídea del tensor. El lector puede encontrar los detalles de su derivación en el trabajo de Idiart \& Ponte Castañeda (2007a), Sección 4b.

Por lo tanto, para calcular la cota externa (3.15) debemos determinar el potencial de tensiones efectivo $\widetilde{u}_{0}$ en términos de la estadística de $\chi^{(r)}$ y los tensores de fase de flexibilidad $\mathbb{S}^{(r)}$. En vista de la linearidad local, este potencial puede ser escrito como

$$
\widetilde{u}_{0}\left(\overline{\boldsymbol{\sigma}} ; \mathbb{S}^{(s)}\right)=\frac{1}{2} \overline{\boldsymbol{\sigma}} \cdot \widetilde{\mathbb{S}}\left(\mathbb{S}^{(s)}\right) \overline{\boldsymbol{\sigma}}
$$

donde $\widetilde{\mathbb{S}}$ es el tensor efectivo de flexibilidad del policristal lineal de comparación. En la práctica, el tensor $\widetilde{\mathbb{S}}$ no puede ser calculado exactamente y debe ser acotado por debajo - en el sentido de formas cuadráticas - de manera tal que el conjunto (3.15) aún acota externamente la superficie de fluencia efectiva de los policristales. Los resultados presentados en este trabajo hacen uso de de la cota inferior de Hashin-Shtrikman derivada por Willis $(1977,1982)$, la cual para policristales porosos toma la forma

$$
\widetilde{\mathbb{S}}=\left[(1-f) \sum_{r=1}^{N} c_{g}^{(r)}\left(\mathbb{S}^{(r)}+\mathbb{S}^{*}\right)^{-1}\right]^{-1}-\mathbb{S}^{*}
$$

donde $\mathbb{S}^{*}=\mathbb{Q}_{0}^{-1}-\mathbb{S}_{0}$ es el tensor de constricción introducido por Hill (1965), $\mathbb{S}_{0}$ es un tensor de flexibilidad de referencia, y $\mathbb{Q}_{0}$ es el tensor microestructural que depende de $\mathbb{S}_{0}$ y de la forma de las funciones de correlación de segundo orden $p^{(r s)}\left(\mathbf{x}, \mathbf{x}^{\prime}\right)$ para la distribución de orientación de granos dentro del agregado - se refiere al lector a Willis (1977,1982) para más detalles. Entonces, esta cota depende de estadísticas microestructurales puntuales de primer y segundo orden y son cotas internas respecto a las cotas 
elementales correspondientes. El tensor de referencia $\mathbb{S}_{0}$ debe ser elegido tal que la desigualdad $\mathbb{S}^{(r)}-\mathbb{S}_{0} \geq 0$ se mantenga para $r=1, \ldots, N$. Nótese que los tensores óptimos $\mathbb{S}^{(r)}$ deben ser incompresibles; una simple elección es

$$
\mathbb{S}_{0}=\frac{1}{2 \mu_{0}} \mathbb{K}
$$

donde $\mathbb{K}$ es el tensor identidad incompresible estándar de cuarto orden y $\left(2 \mu_{0}\right)^{-1}$ se considera como el menor autovalor de todos los tensores $\mathbb{S}^{(r)}$. Con la elección (3.18), la superficie (3.15) acota externamente la superficie de fluencia para todos los policristales con estadística de primer y segundo orden prescripta. El resultado (3.17) también sirve para generar cotas para subclases de policristales con una estadística prescripta; eligiendo

$$
\mathbb{S}_{0}=\widetilde{\mathbb{S}}
$$

el $\widetilde{\mathbb{S}}$ resultante reproduce exactamente la estimación llamada Autoconsistente. Esta estimación es particularmente precisa para sólidos policristalinos como los considerados en este trabajo — véase, por ejemplo, Lebensohn et al. (2004)—, pero más importante aún, es conocido que es exacta para una sub-clase especial de policristales con una estadística prescripta de primer y segundo órden, la cual consiste en microestructuras jerárquicas ${ }^{2}$ con una amplia separación de longuitud de escalas. Por lo tanto, la superficie resultante (3.15) acota externamente el domio elástico de todos los policristales que pertenecen a esa sub-clase particular — véase Nebozhyn et al. (2001).

El cálculo de las funciones $v^{(r)}$ require la solución del problema de optimización (3.14). Como los conjuntos $P^{(r)}$ son polihedros convexos cerrados, formados por el conjunto de planos (o caras) cuyas ecuaciones están dadas por las igualdades (2.1), el máximo en (3.14) es siempre alcanzado en los vértices de dichos polihedros. El conjunto de vértices para cada sistema cristalino se presentó en la sección 2.2 y su determinación ha sido discutida en Idiart (2012) presentándose un breve resúmen en el Anexo A.

\footnotetext{
${ }^{2}$ en inglés se utiliza la palabra hierarchical, hace referencia a un conjunto sucesivo de representaciones de la microestructura cada vez más pequeños en el mismo elemento de volúmen representativo donde la concentración de cada clase de dominio determinada por una orientación espacial determinada, permanece constante
} 


\subsubsection{Relajaciones}

El cálculo de la cota (3.15) puede ser simplificado restringiendo el conjunto de tensores $\mathbb{S}^{(r)}$ a aquellos de la forma

$$
\mathbb{S}^{(r)}=2 \sum_{k=1}^{K} \alpha_{(k)}^{(r)} \boldsymbol{\mu}_{(k)}^{(r)} \otimes \boldsymbol{\mu}_{(k)}^{(r)}, \quad \alpha_{(k)}^{(r)} \geq 0
$$

donde $\boldsymbol{\mu}_{(k)}^{(r)}$ son los tensores de Schmid de la fase cristalina $r$ y las variables escalares $\alpha_{(k)}^{(r)}$ representan las flexibilidades de los sistemas de deslizamiento. Esta clase de tensores de flexibilidad surge naturalmente en las cotas de comparación lineal de deBotton \& Ponte Castañeda (1995); ellas facilitan el cálculo de la función $v$ y la optimización con respecto a las propiedades lineales de comparación. Como resultado de la restricción (3.20) obtenemos una cota débil ${ }^{3} \partial \widetilde{P}_{+}^{\prime}$ tal que $\widetilde{P}_{+} \subset \widetilde{P}_{+}^{\prime}$. Nos referiremos a ella como relajada .

Una segunda simplificación resulta del uso de la desigualdad

$$
v^{(r)}\left(\mathbb{S}^{(r)}\right)=\sup _{\boldsymbol{\sigma} \in P^{(r)}} \frac{1}{2} \boldsymbol{\sigma} \cdot \mathbb{S}^{(r)} \boldsymbol{\sigma} \leq \sum_{k=1}^{K} \alpha_{(k)}^{(r)}\left(\tau_{0}^{(k)}\right)^{2} \doteq v^{(r)^{\prime \prime}}\left(\alpha_{(k)}^{(r)}\right)
$$

para reemplazar las funciones $v$ en $(3.15)$ por la función relajada $v^{\prime \prime}$ similarmente definida en términos de las funciones $v^{(r)^{\prime \prime}}$ presentadas anteriormente; el resultado se puede escribir como

$$
\partial \widetilde{P}_{+}^{\prime \prime}=\left\{\overline{\boldsymbol{\sigma}}: \overline{\boldsymbol{\sigma}}=\Lambda \overline{\boldsymbol{\Sigma}} \text { with }\|\overline{\boldsymbol{\Sigma}}\|=1 \text { and } \Lambda=\inf _{\alpha_{(k)}^{(r)} \geq 0}\left(\frac{\widetilde{u}\left(\overline{\boldsymbol{\Sigma}} ; \alpha_{(k)}^{(s)}\right)}{v^{\prime \prime}\left(\alpha_{(k)}^{(s)}\right)}\right)^{-1 / 2}\right\} .
$$

El sentido de la desigualdad implica que la cota resultante $\partial \widetilde{P}_{+}^{\prime \prime}$ es tal que $\widetilde{P}_{+} \subset \widetilde{P}_{+}^{\prime} \subset$ $\widetilde{P}_{+}^{\prime \prime}$. Esta cota completamente relajada s precisamente la cota derivada originalmente siguiendo una ruta diferente - por deBotton \& Ponte Castañeda (1995). Nótese que la desigualdad en (3.21) se vuelve una igualdad cuando el número total de sistemas de deslizamiento a nivel de cristal simple es cinco y todos ellos son linealmente independientes. En ese caso, las cotas $\partial \widetilde{P}_{+}^{\prime}$ у $\partial \widetilde{P}_{+}^{\prime \prime}$ coinciden.

\footnotetext{
${ }^{3}$ Significa que acota por afuera la cota anterior originando una superficie de fluencia mayor.
} 


\subsection{Cotas de resistencia plástica hidrostática de policristales porosos isótropos}

En esta sección reportamos cotas de Hashin-Shtrikman y Autoconsistentes utilizando el método del medio lineal de comparación para la obtención de la resistencia plástica de sólidos policristalinos con cavidades presurizadas que poseen una simetría isótropa global sujetos a cargas puramente hidrostáticas. Estas condiciones son de especial interés teórico debido a que permiten el tratamiento analítico de las cotas.

Con el fin de estimar el impacto de las relajaciones variacionales de las cotas resultantes se aplican las cotas no relajadas y completamente relajadas restringiendo nuestra atención a sistemas cúbicos y hexagonales con $\tau_{0}^{(k)}=\tau_{0}$ para todos los planos de deslizamiento.

\subsubsection{Aplicación a sólidos cúbicos policristalinos}

\section{Cotas no relajadas}

La resistencia hidrostática $\overline{\boldsymbol{\sigma}}_{h}$ que pertenece a la cota de superficie de fluencia (3.15) está dada por

$$
\overline{\boldsymbol{\sigma}}_{h}=\bar{\sigma}_{h} \mathbf{I}=\Lambda_{h} \overline{\boldsymbol{\Sigma}}_{h} \quad \text { con } \quad \overline{\boldsymbol{\Sigma}}_{h}=\frac{1}{\sqrt{3}} \mathbf{I} \quad \text { y } \quad \Lambda_{h}=\inf _{\mathbb{S}^{(r) \geq 0}}\left(\frac{\widetilde{u}\left(\overline{\boldsymbol{\Sigma}}_{h} ; \mathbb{S}^{(s)}\right)}{v\left(\mathbb{S}^{(s)}\right)}\right)^{-1 / 2},
$$

donde $\bar{\sigma}_{h}$ es una cota superior para la resistencia hidrostática. Debido a la simetría esférica global de la microestructura y la carga, el policristal lineal de comparación debe ser isótropo y los tensores de flexibilidad óptimos $\mathbb{S}^{(r)}$ deben ser $\mathbf{Q}^{(r)}$-rotaciones de un solo tensor $\hat{\mathbb{S}}$; por lo tanto, podemos escribir

$$
\widetilde{u}\left(\bar{\Sigma}_{h} ; \hat{\mathbb{S}}\right)=\frac{1}{6 \widetilde{\kappa}(\hat{\mathbb{S}})} \quad \text { y } \quad v(\hat{\mathbb{S}})=(1-f) \hat{v}(\hat{\mathbb{S}})
$$

donde $\hat{v}$ está dado por la expresión (3.14) con $P^{(r)}$ reemplazado por $\hat{P}$, y $\widetilde{\kappa}$ es el módulo de compresibilidad del policristal lineal de comparación. La expresión para $\widetilde{\kappa}$ depende de la elección específica del método de homogeneización lineal. En esta sección se consideran 
las metodologías para la obtención de cotas de Hashin-Shtrikman (HS) y Autoconsistentes ( $\mathrm{SC}$ en sus siglas en inglés Self-Consistent) presentadas en secciones anteriores. Al especializarlas en el policristal lineal de comparación se llega a la expresión

$$
\widetilde{\kappa}(\hat{\mathbb{S}})=\frac{4}{3} \frac{1-f}{f} \mu_{0}(\hat{\mathbb{S}}),
$$

donde $\mu_{0}$ es el módulo de corte de referencia dictado por las prescripciones

$$
\mu_{0}(\hat{\mathbb{S}})= \begin{cases}\frac{1}{2}\left\|\hat{\mathbb{S}}^{-1}\right\|_{2} & \text { HS } \\ \widetilde{\mu}(\hat{\mathbb{S}}): \quad \mathbb{K} \cdot\left[\mathbb{K}+2 \widetilde{\mu} \frac{3-f}{2+f} \hat{\mathbb{S}}\right]^{-1}-\frac{2+f}{1-f}=0 & \text { SC }\end{cases}
$$

En estas expresiones, $\widetilde{\mu}$ representa el módulo de corte efectivo del policristal lineal de comparación, $\mathbb{K}$ es el tensor de proyección incompresible estándar de cuarto orden, y la norma $\|\cdot\|_{2}$ es el máximo autovalor del tensor. Debido a la simetría cúbica de los cristales, el tensor óptimo $\widehat{\mathbb{S}}$ debe ser de la forma

$$
\hat{\mathbb{S}}=\frac{1}{2 \mu_{a}} \mathbb{K}_{a}+\frac{1}{2 \mu_{s}} \mathbb{K}_{s}=\frac{1}{2 \mu_{s}}\left[\frac{1}{r} \mathbb{K}_{a}+\mathbb{K}_{s}\right]
$$

donde $r \doteq \mu_{a} / \mu_{s}$ es la tasa de anisotropía cúbica y los tensores $\mathbb{K}_{a}$ y $\mathbb{K}_{s}$ denotan los tensores de proyección cúbicos, incompresibles de cuarto orden, véase Walpole (1981), tal que:

$$
\mathbb{K}_{a}=\mathbb{K}_{1}+\mathbb{K}_{2} \quad \text { y } \quad \mathbb{K}_{s}=\mathbb{K}-\mathbb{K}_{a}
$$

El tensor de proyección hidrostática incompresible de cuarto orden $\mathbb{K}$ se puede descomponer en la suma

$$
\mathbb{K}=\mathbb{K}_{1}+\mathbb{K}_{2}+\mathbb{K}_{3}+\mathbb{K}_{4}+\mathbb{K}_{5},
$$

donde los $\mathbb{K}_{n}$ representan los tensores de cuarto orden en el espacio de cinco dimensiones definidos por los siguientes productos diádicos de tensores de segundo orden en la base de Woo (véase el Anexo A para más detalle).

Para esta clase de tensores de flexibilidad, las funciones $\hat{v}$ pueden ser escritas respectivamente como

$$
\hat{v}(\hat{\mathbb{S}})=\frac{\tau_{0}^{2}}{2 \mu_{s}} \operatorname{máx}\left\{\frac{1}{\boldsymbol{\sigma} \in \hat{P}}\left(\tau_{0}^{-1} \boldsymbol{\sigma}\right) \cdot\left[\frac{1}{r} \mathbb{K}_{a}+\mathbb{K}_{s}\right]\left(\tau_{0}^{-1} \boldsymbol{\sigma}\right)\right\} \doteq \frac{\tau_{0}^{2}}{2 \mu_{s}} \hat{v}(r)
$$


mientras que las prescripciones (3.26) para el módulo de referencia pueden ser escritas como $\mu_{0}=m_{0}(r, f) \mu_{s}$, siendo

$$
m_{0}(r, f)= \begin{cases}\operatorname{máx}\{1, r\} & \text { HS } \\ \frac{1-4 f-3 f r+\sqrt{(1-4 f)^{2}+6(4-7 f) r+9 f^{2} r^{2}}}{2(3-f)} & \text { SC }\end{cases}
$$

Introduciendo estas expresiones en (3.23) obtenemos

$$
\frac{\bar{\sigma}_{h}}{\tau_{0}}=\frac{2}{\sqrt{3}} \frac{1-f}{\sqrt{f}} \sqrt{g(f)}
$$

donde

$$
g(f)=\inf _{r \geq 0}\left[m_{0}(r, f) \hat{v}(r)\right]
$$

\section{Cotas completamente relajadas}

Las tensiones hidrostáticas $\overline{\boldsymbol{\sigma}}_{h}$ que pertenecen a la superficie de fluencia acotada (3.22) están dadas por una expresión análoga a (3.23) con la función $v\left(\mathbb{S}^{(s)}\right)$ reemplazada por $v^{\prime \prime}\left(\alpha_{(k)}^{(s)}\right)$. Una vez más, debido a la simetría esférica de la microestructura y de las cargas, el policristal lineal de comparación debe ser isótropo y los tensores de flexibilidad asociados con el conjunto de $\alpha_{(k)}^{(r)}$ óptimos deben ser todos $\mathbf{Q}^{(r)}$ rotaciones de un solo tensor de flexibilidad con simetría cúbica. Las cotas de Hashin-Shtrikman y Autoconsistentes pueden ser obtenidas por caminos análogos a aquellos de la sección previa; el resultado puede ser escrito como (3.32), donde

$$
g(f)=\inf _{\alpha_{(k)} \geq 0}\left[m_{0}(r, f) \hat{v}^{\prime \prime}\left(\alpha_{(k)}\right)\right] .
$$

En esta expresión, para cristales cúbicos $m_{0}(r, f)$ es la misma función $(3.31), \hat{v}^{\prime \prime} \doteq$ $2 \mu_{s} v^{\prime \prime} / \tau_{0}^{2}$, donde

$$
r=\frac{2}{3} \frac{\sum_{k=1}^{K} \alpha_{(k)} \boldsymbol{\mu}_{(k)} \cdot \mathbb{K}_{s} \boldsymbol{\mu}_{(k)}}{\sum_{k=1}^{K} \alpha_{(k)} \boldsymbol{\mu}_{(k)} \cdot \mathbb{K}_{a} \boldsymbol{\mu}_{(k)}} \quad \text { y } \quad \hat{v}^{\prime \prime}\left(\alpha_{(k)}\right)=\frac{3}{2} \frac{\sum_{k=1}^{K} \alpha_{(k)}}{\sum_{k=1}^{K} \alpha_{(k)} \boldsymbol{\mu}_{(k)} \cdot \mathbb{K}_{s} \boldsymbol{\mu}_{(k)}} .
$$

La estructura matemática de la función objetivo en (3.34) implica que las flexibilidades óptimas de deslizamiento $\alpha_{(k)}$ asociadas con todos los sistemas de deslizamiento con 
iguales projecciones $\boldsymbol{\mu}_{(k)} \cdot \mathbb{K}_{s} \boldsymbol{\mu}_{(k)}$ y $\boldsymbol{\mu}_{(k)} \cdot \mathbb{K}_{a} \boldsymbol{\mu}_{(k)}$ tomarán los mismos valores. Nótese también que la función objetivo es homogénea de grado cero en las flexibilidades de deslizamiento.

\subsubsection{Aplicación a sólidos hexagonales policristalinos}

\section{Cotas no relajadas}

Las ecuaciones (3.23) a (3.26) siguen siendo válidas mientras que debido a la simetría cristalina hexagonal, el tensor óptimo $\widehat{\mathbb{S}}$ debe ser de la forma

$$
\hat{\mathbb{S}}=\frac{1}{2 \mu_{b}} \mathbb{K}_{b}+\frac{1}{2 \mu_{c}} \mathbb{K}_{c}+\frac{1}{2 \mu_{d}} \mathbb{K}_{d}=\frac{1}{2 \mu_{b}}\left[\mathbb{K}_{b}+\frac{1}{r_{c}} \mathbb{K}_{c}+\frac{1}{r_{d}} \mathbb{K}_{d}\right],
$$

donde $r_{c} \doteq \mu_{c} / \mu_{b}$ y $r_{d} \doteq \mu_{d} / \mu_{b}$ son las tasas de anisotropía hexagonales y los tensores $\mathbb{K}_{b}=\mathbb{K}_{1}, \mathbb{K}_{c}=\mathbb{K}_{2}+\mathbb{K}_{3}$ y $\mathbb{K}_{d}=\mathbb{K}_{4}+\mathbb{K}_{5}$. Para esta clase de tensores de flexibilidad, la función $\hat{v}$ puede ser escrita como

$$
\hat{v}(\hat{\mathbb{S}})=\frac{\tau_{0}^{2}}{2 \mu_{b}} \operatorname{máx}\left\{\frac{1}{\boldsymbol{\sigma} \in \hat{P}}\left(\tau_{0}^{-1} \boldsymbol{\sigma}\right) \cdot\left[\mathbb{K}_{b}+\frac{1}{r_{c}} \mathbb{K}_{c}+\frac{1}{r_{d}} \mathbb{K}_{d}\right]\left(\tau_{0}^{-1} \boldsymbol{\sigma}\right)\right\} \doteq \frac{\tau_{0}^{2}}{2 \mu_{b}} \hat{v}\left(r_{c}, r_{d}\right),
$$

mientras que las prescripciones (3.26) para el módulo de referencia pueden ser escritas como $\mu_{0}=m_{0}\left(r_{t}, f\right) \mu_{b}$ siendo

$$
m_{0}\left(r_{t}, f\right)= \begin{cases}\operatorname{máx}\left\{1, r_{t}\right\} & \text { HS } \\ \frac{1-4 f-3 f r_{t}+\sqrt{(1-4 f)^{2}+6(4-7 f) r_{t}+9 f^{2} r_{t}^{2}}}{2(3-f)} & \text { SC }\end{cases}
$$

Nótese que en la expresión (3.38) $m_{0}=m_{0}\left(r_{t}, f\right)$. Esto se debe a que se observó numéricamente que el problema de optimización (3.40) tiene solución para la elección $r_{c}=r_{d}=r_{t}$. Introduciendo estas expresiones en (3.23) obtenemos

$$
\frac{\bar{\sigma}_{h}}{\tau_{0}}=\frac{2}{\sqrt{3}} \frac{1-f}{\sqrt{f}} \sqrt{g(f)}
$$

donde

$$
g(f)=\inf _{r_{t} \geq 0}\left[m_{0}\left(r_{t}, f\right) \hat{v}\left(r_{t}\right)\right]
$$




\section{Cotas completamente relajadas}

Nuevamente las tensiones hidrostáticas $\overline{\boldsymbol{\sigma}}_{h}$ que pertenecen a la superficie de fluencia acotada (3.22) están dadas por una expresión análoga a (3.23) con las funciones $v\left(\mathbb{S}^{(s)}\right)$ reemplazadas por $v^{\prime \prime}\left(\alpha_{(k)}^{(s)}\right)$. Una vez más, debido a la simetría esférica de la microestructura y de las cargas, el policristal lineal de comparación debe ser isótropo y los tensores de flexibilidad asociados con el conjunto de $\alpha_{(k)}^{(r)}$ óptimos deben ser todos $\mathbf{Q}^{(r)}$ rotaciones de un simple tensor de flexibilidad con simetría hexagonal. Las cotas de Hashin-Shtrikman y Autoconsistentes pueden ser obtenidas por caminos análogos a aquellos de la sección previa; el resultado puede ser escrito como (3.32) donde

$$
g(f)=\inf _{r_{c}, r_{d} \geq 0}\left[m_{0}\left(r_{c}, r_{d}, f\right) \hat{v}^{\prime \prime}\left(r_{c}, r_{d}\right)\right] .
$$

En esta expresión, para cristales hexagonales $m_{0}\left(r_{t}, f\right)$ es la misma función $(3.38), \hat{v}^{\prime \prime} \doteq$ $2 \mu_{b} v^{\prime \prime} / \tau_{0}^{2}$, donde

$$
\begin{gathered}
r_{d}=2 \frac{\sum_{k=1}^{K} \alpha_{(k)} \boldsymbol{\mu}_{(k)} \cdot \mathbb{K}_{b} \boldsymbol{\mu}_{(k)}}{\sum_{k=1}^{K} \alpha_{(k)} \boldsymbol{\mu}_{(k)} \cdot \mathbb{K}_{d} \boldsymbol{\mu}_{(k)}} \quad ; \quad r_{c}=2 \frac{\sum_{k=1}^{K} \alpha_{(k)} \boldsymbol{\mu}_{(k)} \cdot \mathbb{K}_{b} \boldsymbol{\mu}_{(k)}}{\sum_{k=1}^{K} \alpha_{(k)} \boldsymbol{\mu}_{(k)} \cdot \mathbb{K}_{c} \boldsymbol{\mu}_{(k)}} \quad \mathrm{y} \\
\hat{v}^{\prime \prime}\left(\alpha_{(k)}\right)=\frac{\sum_{k=1}^{K} \alpha_{(k)}}{2 \sum_{k=1}^{K} \alpha_{(k)} \boldsymbol{\mu}_{(k)} \cdot \mathbb{K}_{b} \boldsymbol{\mu}_{(k)}} .
\end{gathered}
$$

Como en el caso cúbico, la estructura matemática de la función objetivo en (3.41) implica que las flexibilidades óptimas de deslizamiento $\alpha_{(k)}$ asociadas con todos los sistemas de deslizamiento con iguales projecciones $\boldsymbol{\mu}_{(k)} \cdot \mathbb{K}_{b} \boldsymbol{\mu}_{(k)}, \boldsymbol{\mu}_{(k)} \cdot \mathbb{K}_{d} \boldsymbol{\mu}_{(k)}$ y $\boldsymbol{\mu}_{(k)} \cdot \mathbb{K}_{c} \boldsymbol{\mu}_{(k)}$ tomarán los mismos valores.

\subsubsection{Resultados para sistemas materiales específicos}

En esta sección reportamos las cotas para los sistemas materiales cúbicos y hexagonales (véase Anexo) presentados en la sección 2.2. Las cotas de Hashin-Shtrikman y Autoconsistentes para los sistemas materiales se obtienen resolviendo problemas de optimización presentados en las secciones anteriores obteniendo de este modo las funciones $g(f)$ : 
FCC: $\quad g(f)= \begin{cases}6 & \mathrm{HS} \\ 9 & \mathrm{HS}^{\prime \prime} \\ \frac{1-5 f+\sqrt{9-22 f+17 f^{2}}}{1-f / 3} & \mathrm{SC} \\ \frac{3}{2} \frac{1-5 f+\sqrt{9-22 f+17 f^{2}}}{1-f / 3} & \mathrm{SC}^{\prime \prime}\end{cases}$

BCC: $\quad g(f)= \begin{cases}3(5-2 \sqrt{3}) & \mathrm{HS} \\ 9 & \mathrm{HS}^{\prime \prime} \\ 3(2-\sqrt{3}) \frac{1-5 f+\sqrt{9-22 f+17 f^{2}}}{1-f / 3} & \mathrm{SC} \\ \frac{3}{2} \frac{1-5 f+\sqrt{9-22 f+17 f^{2}}}{1-f / 3} & \mathrm{SC}^{\prime \prime}\end{cases}$

$(3+$ HS

$\mathrm{HS}^{\prime \prime}$

$\mathrm{HCP}: \quad g(f)= \begin{cases}3 & \mathrm{HS} \\ 5 & \mathrm{HS}^{\prime \prime} \\ 10(2-\sqrt{3}) \frac{1-2 f}{1-f / 3} & \mathrm{SC} \\ 5 \frac{1-2 f}{1-f / 3} & \mathrm{SC}^{\prime \prime}\end{cases}$

$\mathrm{SC}$

$\mathrm{SC}^{\prime \prime}$ 


$$
\begin{aligned}
& \text { IONIC AB: } g(f)= \begin{cases}\frac{10}{3} & \mathrm{HS} \\
5 & \mathrm{HS}^{\prime \prime} \\
\frac{10}{3} \frac{1-2 f}{-1+f / 3} & \mathrm{SC} \\
5 \frac{1-2 f}{1-f / 3} & \mathrm{SC}^{\prime \prime}\end{cases} \\
& (\infty) \text { HS } \\
& \infty \quad \mathrm{HS}^{\prime \prime} \\
& \text { HCP AB: } g(f)=\left\{\begin{array}{l}
\infty \\
\frac{3 \sqrt{3}}{2} \frac{1-2 f}{1+2 f} \frac{2+f}{1-f / 3}
\end{array} \quad\right. \text { SC } \\
& 4 \frac{1-2 f}{1+2 f} \frac{2+f}{1-f / 3} \quad \mathrm{SC}^{\prime \prime} \\
& \text { IONIC B: } g(f)= \begin{cases}\infty & \mathrm{HS} \\
\infty & \mathrm{HS}^{\prime \prime} \\
2 \frac{1-2 f}{f} \frac{2+f}{3-f} & \mathrm{SC} \\
3 \frac{1-2 f}{f} \frac{2+f}{3-f} & \mathrm{SC}^{\prime \prime}\end{cases}
\end{aligned}
$$

donde los identificadores no primados y las doblemente primados se refieren cotas no relajadas y completamente relajadas, respectivamente. Las cotas Autoconsistentes son válidas en el rango de porosidades $0 \leq f \leq 1 / 2$. La cota Autoconsistente para materiales IONIC dada en (3.46) y (3.47) no es exacta, pero es un resultado aproximado con alto grado de precisión resultando conveniente a fines comparativos. 



\section{Capítulo 4}

\section{Resultados para policristales}

\section{isótropos cúbicos y hexagonales}

Las cotas lineales de comparación presentadas en el capítulo anterior se aplican a varias clases de sólidos policristalinos isótropos y porosos. En todos los casos, se asume que tanto la textura morfológica como la cristalográfica son estadísticamente isótropas, por lo tanto los agregados exhiben una isotropía plástica global. Esto es equivalente a asumir que las funciones de correlación de segundo orden $p^{(r s)}$ son isótropas y que $c_{g}^{(r)}=1 / N(r, s=1, \ldots, N)$. En vista de la isotropía global, la superficie de fluencia efectiva puede ser expresada en términos de los tres invariantes isótropos del tensor de tensiones $\bar{\sigma}_{m}, \bar{\sigma}_{e} \mathrm{y} \bar{\theta}$ definidos por

$$
\bar{\sigma}_{m}=\frac{1}{3} \operatorname{tr} \overline{\boldsymbol{\sigma}}, \quad \bar{\sigma}_{e}=\sqrt{\frac{3}{2} \overline{\boldsymbol{\sigma}}_{d} \cdot \overline{\boldsymbol{\sigma}}_{d}}, \quad \mathrm{y} \quad \cos (3 \bar{\theta})=\frac{27}{2} \operatorname{det}\left(\frac{\overline{\boldsymbol{\sigma}}_{d}}{\bar{\sigma}_{e}}\right),
$$

donde $\overline{\boldsymbol{\sigma}}_{d}$ es la parte desviadora de $\overline{\boldsymbol{\sigma}}$. El invariante de tensiones $\bar{\theta}$ es una función homogénea de grado cero en $\overline{\boldsymbol{\sigma}}$ y caracteriza la 'dirección' de las tensiones de corte macroscópicas en el espacio desviador: los valores particulares $\bar{\theta}=0$ y $\bar{\theta}=\pi / 6$ corresponden a cargas de corte axisimétrico y simple, respectivamente. Sin embargo, la variación de $\widetilde{\sigma}_{0}$ con $\bar{\theta}$ no es estudiada en este trabajo, únicamente se considera el caso de cargas axisimétricas. Por último, se define $\bar{X}_{\sigma}=\bar{\sigma}_{m} / \bar{\sigma}_{e}$ como la tasa de triaxialidad de tensiones.

Los resultados presentados en las secciones siguientes corresponden a doscientas orientaciones cristalinas $(N=200)$ obtenidas mediante el algoritmo de Sobol (Sobol 1967) 
para lograr texturas lo más cercanas a la isotropía — véase, por ejemplo, Lebensohn et al. (2011). De cualquier modo, se utilizó el mismo conjunto de orientaciones para todos los cálculos de tal forma que tenga sentido la comparación entre diferentes cotas. Los distintos algoritmos utilizados para realizar los cálculos están descriptos en Idiart (2012). De aquí en adelante, las cotas no relajadas de Hashin-Shtrikman y Autoconsistentes se designarán por HS y SC, respectivamente, mientras que sus versiones relajadas, con letras mayúsculas doblemente primadas. Las cotas intermedias relajadas no han sido consideradas en este estudio ya que es de esperarse que sean muy cercanas a las cotas no-relajadas.

\subsection{Policristales cúbicos}

Se reportan resultados para tres tipos de sólidos policristalinos: FCC, BCC y IONIC.

La figura 4.1 muestra las cotas para las superficies de fluencia de varios sólidos cúbicos con una resistencia a la fluencia $\tau_{0}$ para todos los sistemas de deslizamiento y moderado nivel de porosidad $(f=0,05)$. Se observa que la cota elemental de Taylor produce una superficie cilíndrica abierta paralela al eje de tensiones hidrostáticas, mientras que las cotas de HS y SC producen superficies suaves, cerradas y convexas dentro de la superficie de Taylor, como es de esperarse. Nótese que si bien la superficie de fluencia a nivel cristalino posee vértices -ya que es un polihedro formado por facetas determinadas por los hiperplanos dados por la ley de Schmidt-, la isotropía en la textura cristalográfica genera una superficie de fluencia suave sin puntas aún en el caso de grandes triaxialidades.

La principal observación en el contexto de esta figura, sin embargo, es que en todos los casos la relajación variacional produce un detrimento considerable en las cotas lineales de comparación. El impacto global puede ser cuantificado por comparación de las normas de los dominios elásticos encerrados por la resistencia plástica. Se encontró para sólidos cristalinos cúbicos FCC y IONIC un modesto valor de $\sim 15 \%$ pero mayor en el 
a)

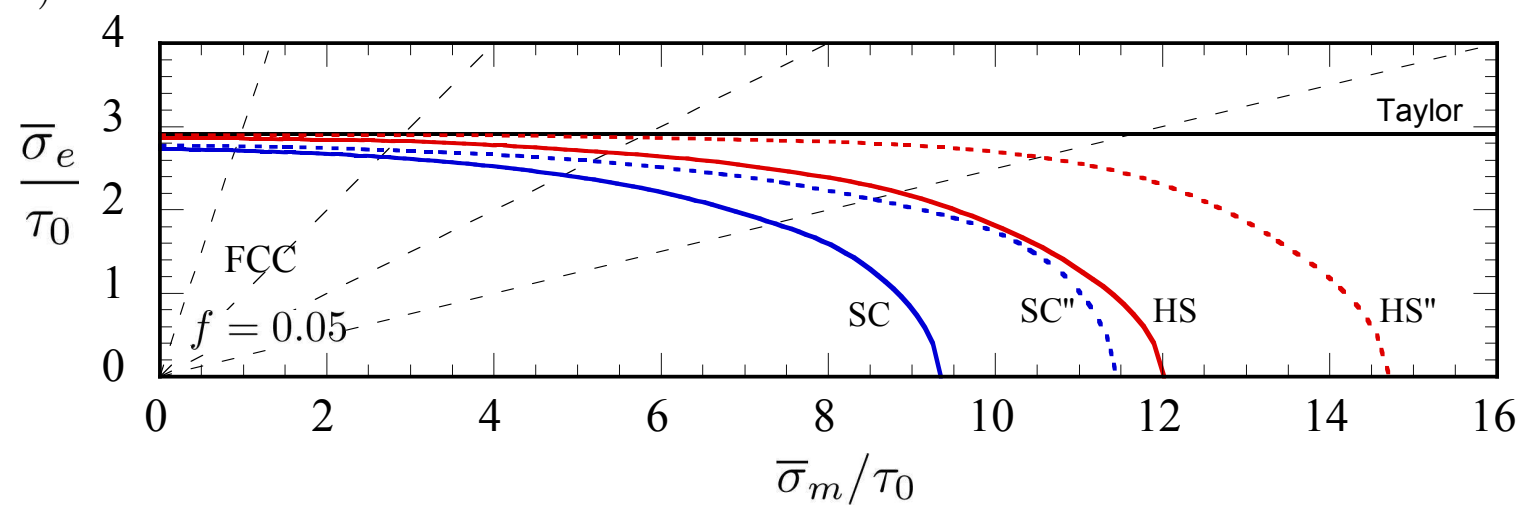

b)

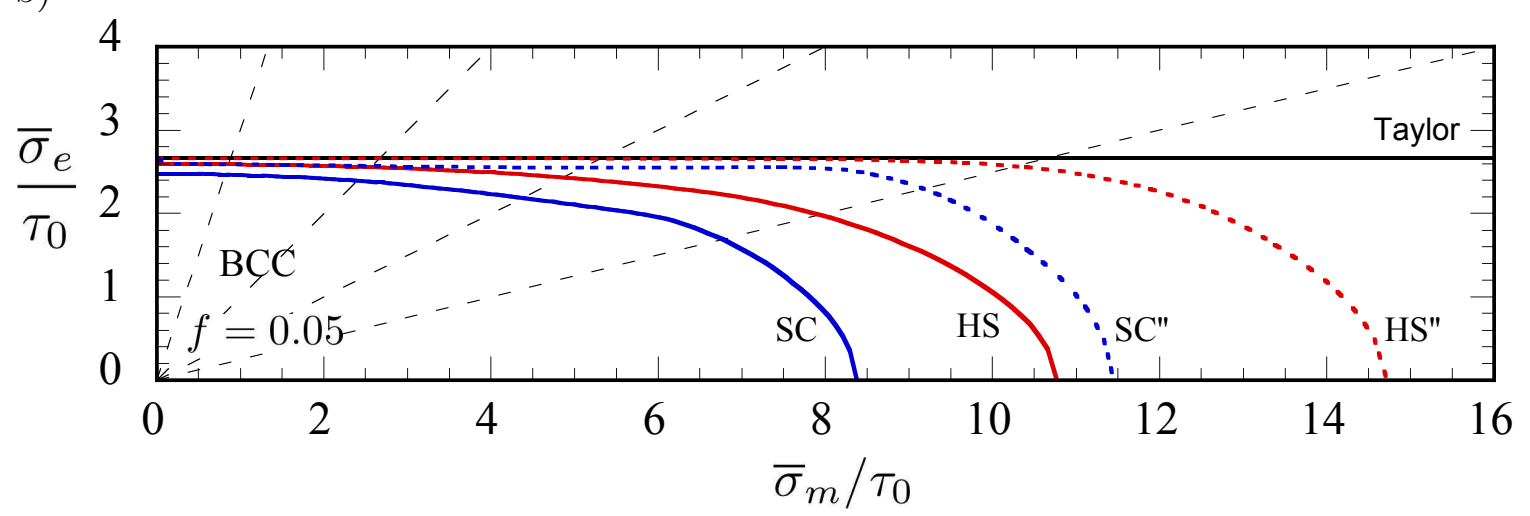

c)

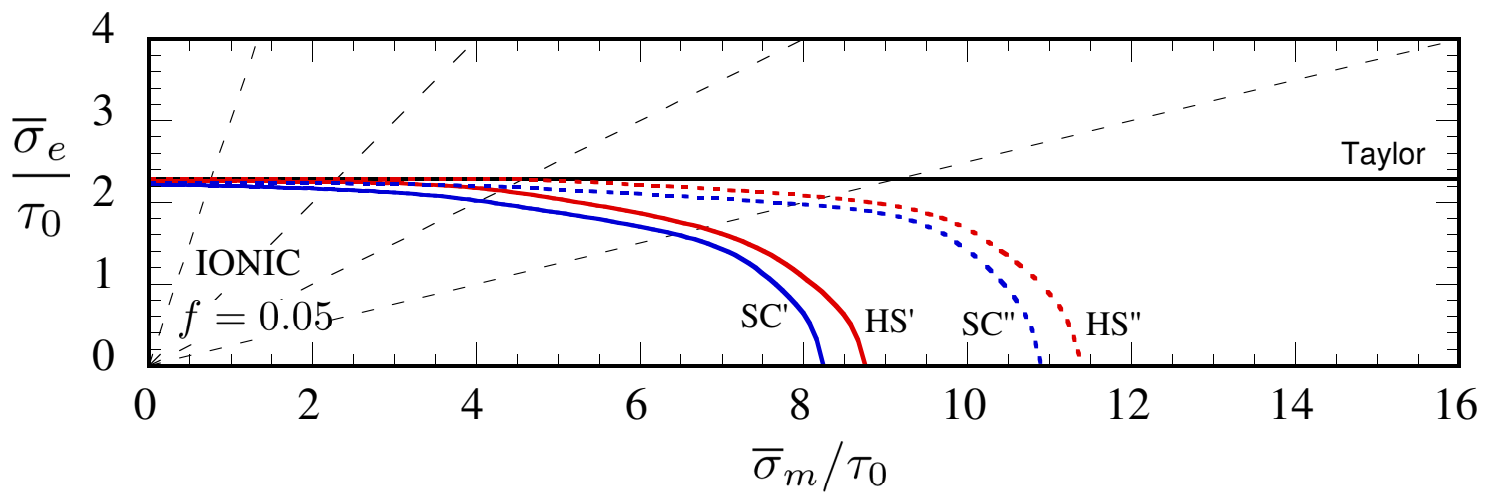

Figura 4.1: Cotas de superficie de fluencia de policristales cúbicos isótropos con moderado nivel de porosidad $(f=0,05)$, sujetos a cargas axisimétricas. Las líneas discontínuas indican direcciones de triaxilidad de tensiones constante $\bar{X}_{\sigma}=1 / 3,1,2,4$. 
caso de los sólidos BCC llegando al $\sim 25 \%^{1}$. Sin embargo, el impacto varía ampliamente con la triaxialidad en tensiones considerada. El impacto observado en las cotas para la resistencia al corte es menor al $2 \%$ en sólidos FCC y BCC, del $\sim 7 \%$ en sólidos IONIC, mientras que para la resistencia hidrostática es del $\sim 22 \%$ en sólidos FCC, $\sim 29 \%$ en sólidos IONIC y 40\% en sólidos BCC. En todos los casos, el impacto es significativamente mayor que el observado previamente por Idiart (2012) en policristales sólidos sin porosidad. Además, se observa que las diferencias entre las cotas no relajadas correspondientes para FCC y BCC son mayores que aquellas entre sus contrapartes relajadas. Por lo tanto, la relajación pareciera reducir la sensibilidad de las cotas a la cristalinidad de la matriz.

Más notable aún es el hecho de que las cotas no relajadas de HS no relajados son más estrictas que las totalmente relajadas SC" por encima de cierta triaxialidad de tensiones en sólidos IONIC y BCC; este hecho no ha sido observado en el contexto de sistemas sólidos sin porosidad. La metodología de homogeneización de HS provee cotas para una clase entera de policristales, que incluyen la subclase de policristales que dan cuenta del esquema SC. Por lo tanto, los resultados HS no relajados constituyen una cota superior rigurosa para el resto de los resultados incluyendo los SC". Esto es indicativo de que la pérdida resultante por la relajación (3.21) depende más fuertemente de los parámetros materiales tales como la heterogeneidad en el contraste que del número de sistemas de deslizamiento de los cristales del agregado. Recordemos que las técnicas lineales de comparación como las consideradas en este trabajo arrojan predicciones bastante realistas para bajas y moderadas triaxialidades pero predicciones irrealistas bajo grandes triaxialidades — véase, por ejemplo, Lebensohn et al. (2011). Mientras que no se espera que las mejoras significantes halladas en este trabajo provean predicciones realistas para altas triaxialidades, sí se espera que aumenten el rango de triaxialidades para el cual las mismas son precisas.

\footnotetext{
${ }^{1}$ Los porcentajes corresponden a la diferencia entre las cotas relajadas y no relajadas relativos al resultado no relajado.
} 
a)

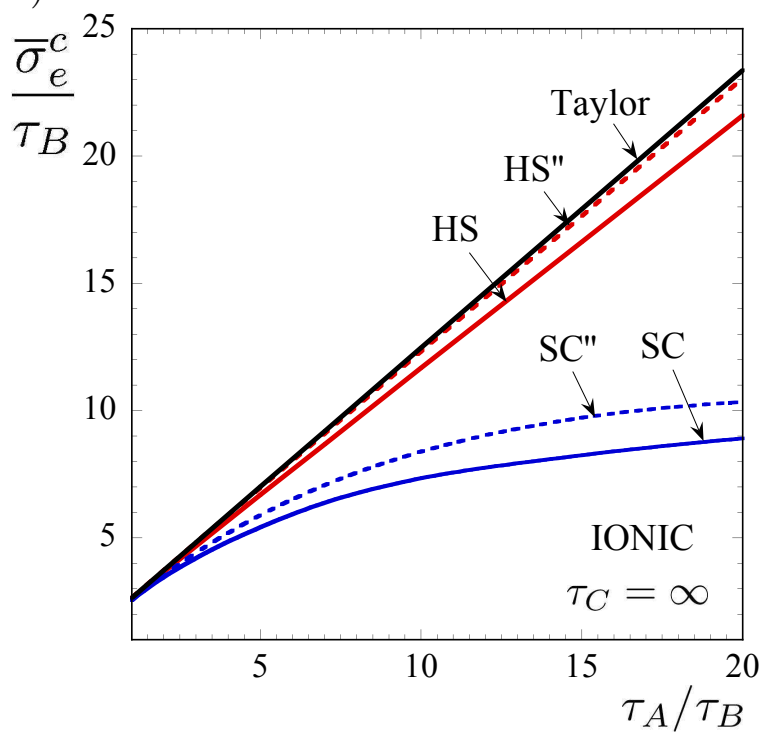

c)

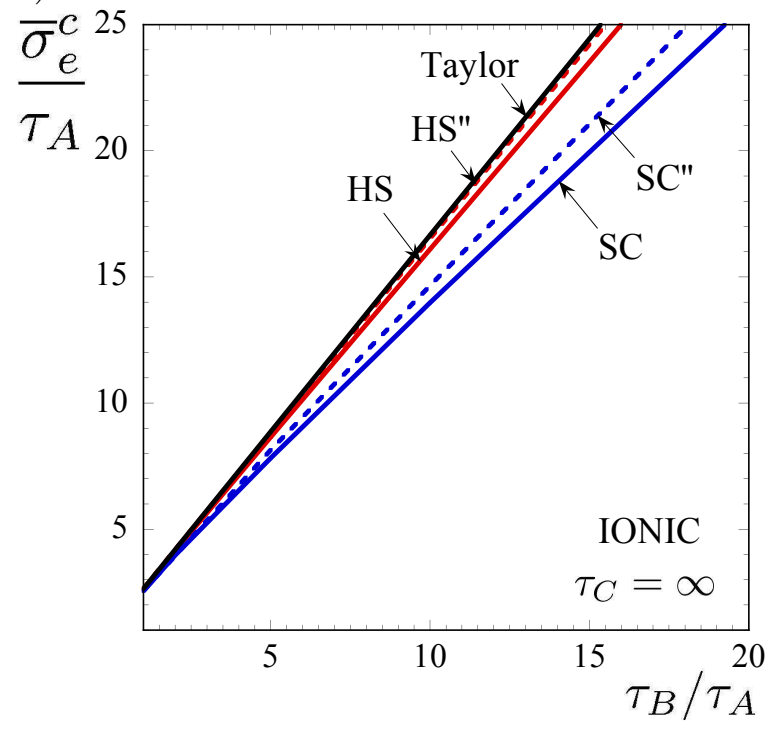

b)

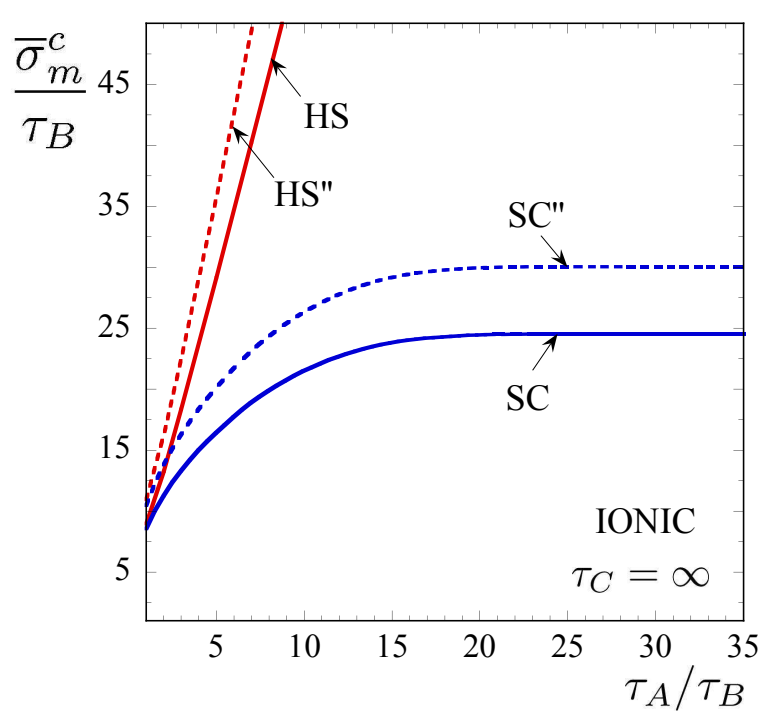

d)

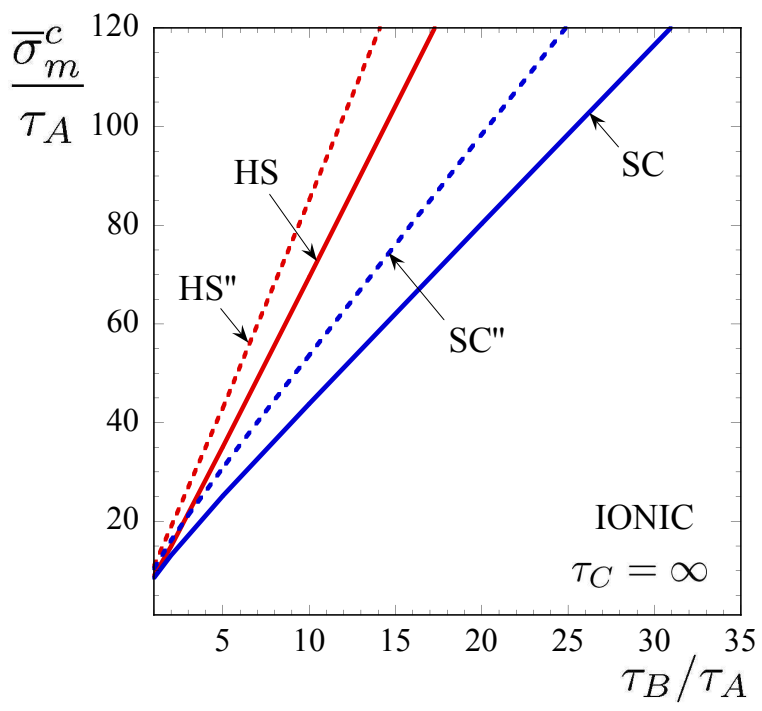

Figura 4.2: Cotas de resistencia plástica efectiva bajo cargas de corte $\left(\bar{\sigma}_{e}^{c}\right)$ e hidrostáticas $\left(\bar{\sigma}_{m}^{c}\right)$ para policristales iónicos (IONIC) isótropos con moderado nivel de porosidad $(f=$ 0,05), como función del contraste en los sistemas de deslizamiento. 
La figura 4.2 muestra varias cotas para sólidos IONIC con grado moderado de porosidad $(f=0,05)$ y varios grados de anisotropía cristalina. Las curvas están dadas para resistencias plásticas de corte $\left(\bar{\sigma}_{e}^{c}\right)$ e hidrostáticas $\left(\bar{\sigma}_{m}^{c}\right)$ como función del contraste entre los sistemas de deslizamiento A y B en sólidos IONIC con sistemas tipo C infinitamente rígidos. Comenzaremos notando que las cotas para la resistencia al corte muestran tendencias similares a las reportadas previamente para sólidos IONIC sin porosidad — véase figura 1 en Idiart (2012) - , como es de esperarse para este grado de porosidad. De este modo, las cotas de Taylor y HS crecen linealmente con el contraste en los sistemas de deslizamiento y se acercan bastante entre ellas para todo el rango de anisotropías plásticas considerado, mientras que las cotas SC divergen de aquellas cotas exhibiendo un crecimiento diferente y grandes diferencias debidas a la relajación variacional. Por otra parte, la cota de Taylor para la resistencia hidrostática es infinitamente grande, y por lo tanto trivial, mientras que las cotas de HS y SC para esta cantidad permanecen finitas siempre y cuando los sistemas de deslizamiento tipo- $A$ y tipo- $B$ puedan ambos deformar. Una vez más, las cotas para la resistencia hidrostática muestran las máximas diferencias relativas debido a la relajación variacional. Sin embargo, esas diferencias se mantienen del mismo orden $(\sim 22 \%)$ que aquellas reportadas anteriormente para sólidos IONIC de gran simetría. Cálculos adicionales en función de la porosidad — que serán presentados en la sección 4.4- revelan que el impacto en la relajación variacional de esas cotas lineales de comparación es independiente del nivel de porosidad. Finalmente, como las cotas de HS crecen más rápido con el contraste que las cotas SC, las cotas no relajadas de HS para la resistencia hidrostática dejan de ser más estrictas que las cotas relajadas SC" por encima de cierto nivel de contraste. 


\subsection{Policristales hexagonales}

En esta sección se presentan los resultados para sólidos policristalinos con simetría hexagonal y una relación de aspecto de $c / a=1,59$. La figura 4.3 muestra las cotas

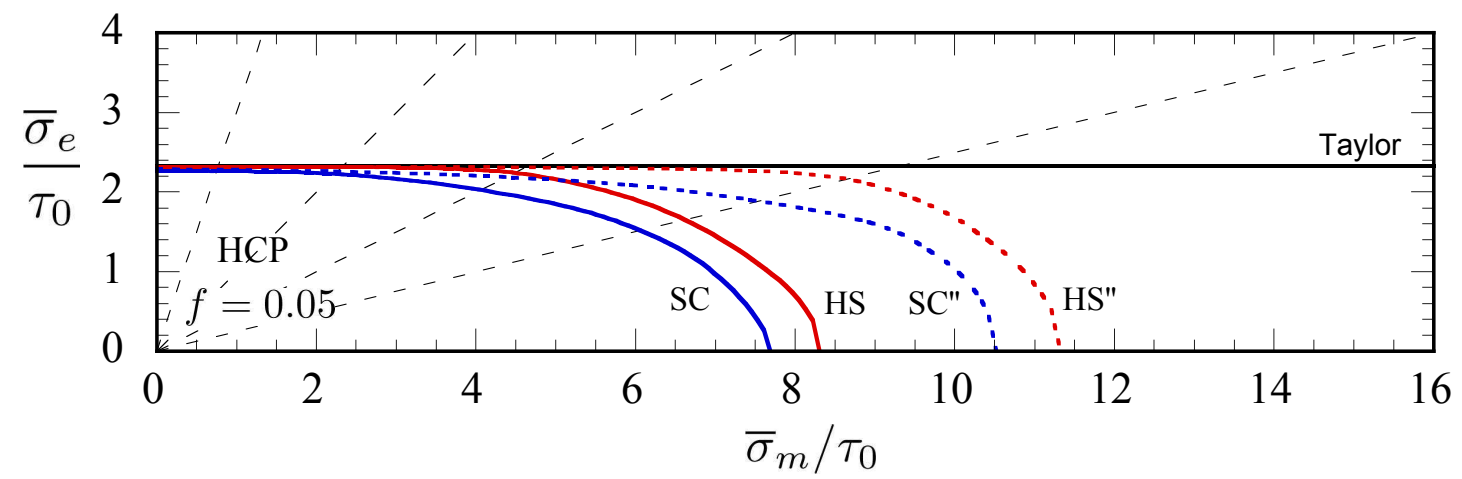

Figura 4.3: Cotas de superficie de fluencia de policristales hexagonales isótropos con un nivel de porosidad moderada $(f=0,05)$, sujetos a cargas axisimétricas. Las líneas discontínuas indican direcciones de triaxialidad de tensiones constante $\bar{X}_{\sigma}=1 / 3,1,2,4$.

para la superficie de fluencia de sólidos hexagonales con $\tau_{A}=\tau_{B}=\tau_{C}=\tau_{0}$ y un nivel moderado de porosidad $(f=0,05)$. Una vez más, las cotas lineales de comparación producen superficies suaves y convexas, contenidas por la superficie de Taylor, como es de esperarse; la relajación de la función $v$ produce un notorio impacto en detrimento de esas superficies, especialmente a grandes triaxialidades en tensión. El impacto global de la relajación ${ }^{2}$ es $\sim 21 \%$, pero el impacto en la resistencia al corte es menos de $1 \%$ mientras que en la resistencia hidrostática es $\sim 40 \%$. Además, las cotas no relajadas de HS son significantemente más estrictas que las cotas relajadas SC" por encima de cierto grado de triaxialidad, como se ha observado en sólidos IONIC y BCC. Sin embargo se hace hincapié en que debido a que las cotas SC muestran un nivel de porosidad de percolación menor respecto a las cotas de HS, ésta deja de ser más estricta que la SC" a grandes niveles de porosidad.

\footnotetext{
${ }^{2}$ Se define como la norma de los dominios elásticos.
} 
La figura 4.4 muestra varias cotas para sólidos $\mathrm{HCP}$ con un moderado nivel de porosidad $(f=0,05)$ y varios grados de anisotropía cristalina. Las figuras se presentan para resistencias plásticas tanto de corte $\left(\bar{\sigma}_{e}^{c}\right)$ como hidrostáticas $\left(\bar{\sigma}_{m}^{c}\right)$ en función del contraste en los sistemas de deslizamiento en sólidos con $\tau_{A}=\tau_{B} \neq \tau_{C}$ y $\tau_{A} \neq \tau_{B}=\tau_{C}$. Una vez más, las cotas para la resistencia al corte muestran tendencias similares a las observadas previamente en sólidos sin porosidad — véase la figura 2 en Idiart (2012)-, como es de esperarse a este nivel de porosidad. Por consiguiente, las cotas de Taylor y HS crecen linealmente con el contraste en los sistemas de deslizamiento y nos brindan predicciones similares, mientras que las cotas SC divergen de aquellas cotas mostrando un crecimiento diferente y brindando predicciones presumiblemente más realistas. Por otra parte, la cota de Taylor para la resistencia hidrostática es infinitamente grande mientras que las cotas de HS y SC para esta cantidad permanecen finitas, siempre y cuando los tres sistemas de deslizamiento puedan deformar. El impacto en la relajación variacional de las cotas lineales de comparación permanece en el orden del $\sim 40 \%$ pero decrece algo al incrementarse el contraste en los sistemas de deslizamiento. De este modo, el efecto de la relajación parece depender de la porosidad, la cristalografía y la anisotropía cristalina de manera intrincada. Finalmente, debido a que las cotas de HS crecen más rápido con el contraste en los sistemas de deslizamiento que las cotas SC, las cotas no relajadas HS para la resistencia hidrostática dejan de ser más estrictas que las cotas relajadas SC" por encima de cierto contraste.

\subsection{Policristales con sistemas de deslizamiento de- ficientes}

Al hacer tender los contrastes en los sistemas de deslizamiento de las figuras 4.2 y 4.4 a infinito, el número de sistemas de deslizamientos linealmente independientes en los correspondientes cristales que constituyen el policristal se vuelve deficiente, es decir, menor a cinco. En este límite, las cotas para las resistencias plásticas de corte e 
a)

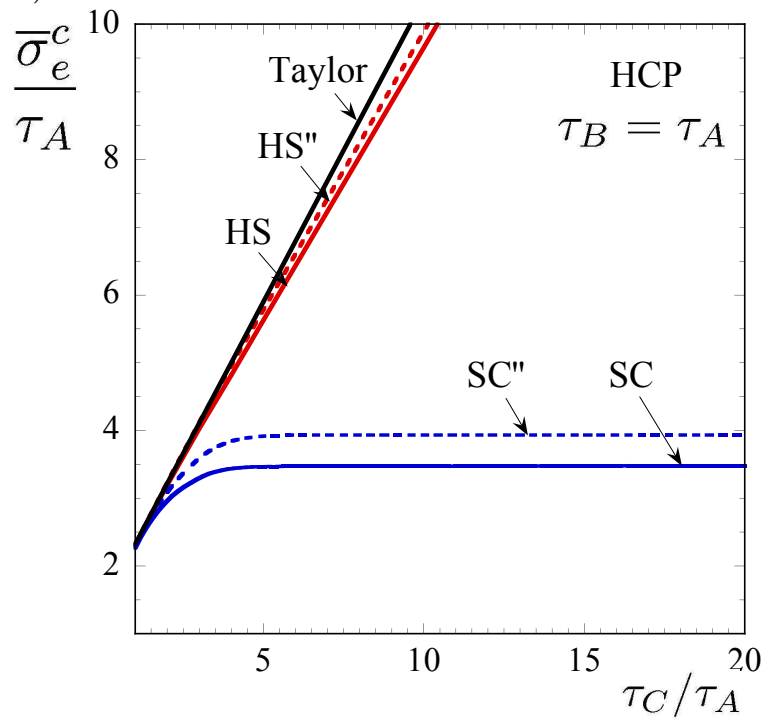

c)

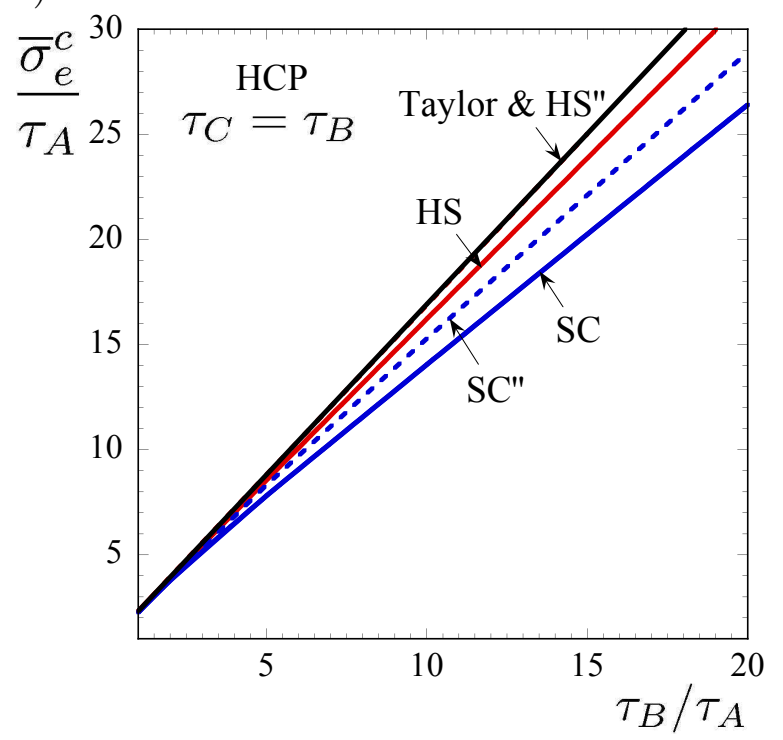

b)

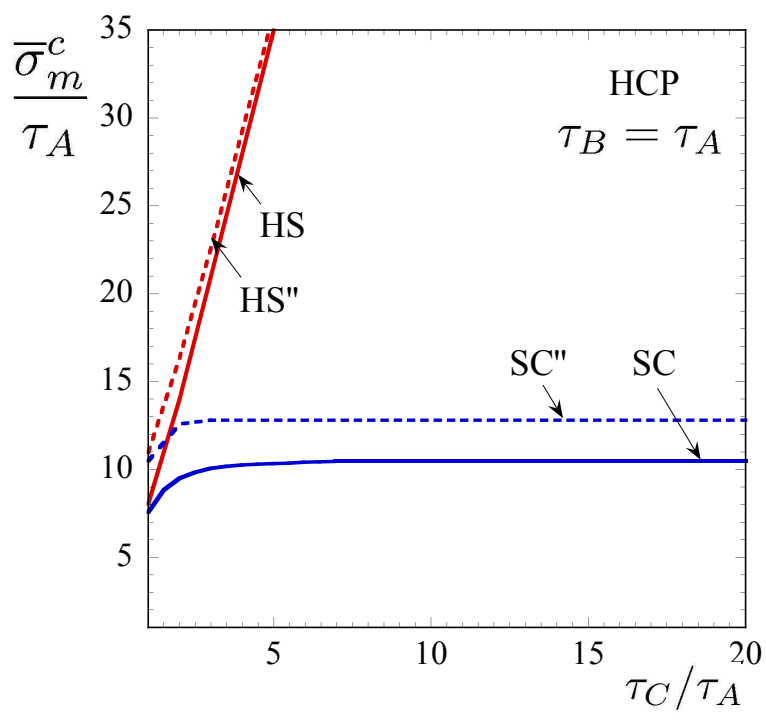

d)

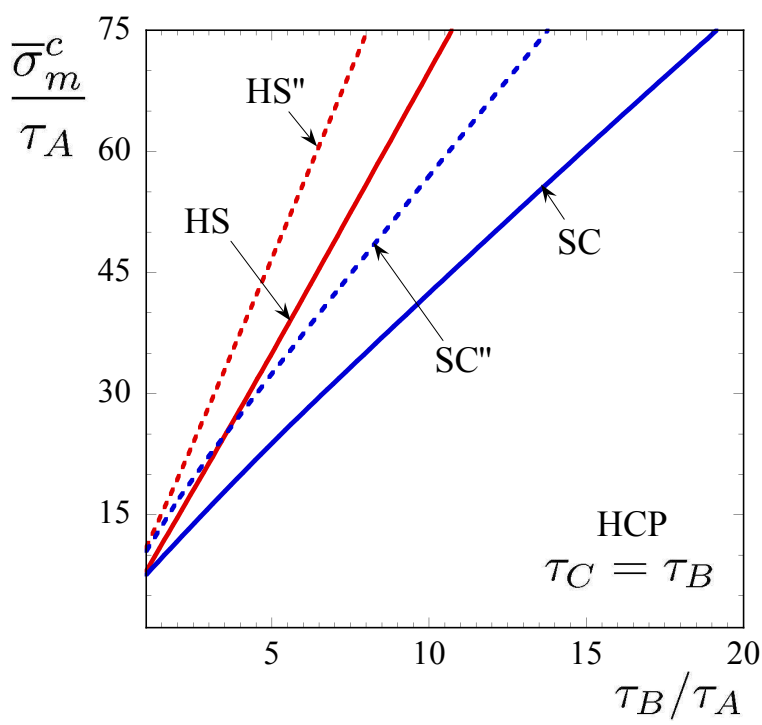

Figura 4.4: Cotas de resistencia plástica efectiva bajo cargas de corte $\left(\bar{\sigma}_{e}^{c}\right)$ e hidrostáticas $\left(\bar{\sigma}_{m}^{c}\right)$ para policristales hexagonales $(\mathrm{HCP})$ isótropos con un nivel de porosidad moderada $f=0,05$, como función del contraste al deslizamiento. 
hidrostáticas siguen un factor de escalamiento de la forma $\bar{\sigma}_{e}^{c} \sim M^{\gamma_{e}}$ y $\bar{\sigma}_{m}^{c} \sim M^{\gamma_{m}}$, donde $M$ es el contraste en sistemas de deslizamiento relevante. Más específicamente, para la cota de Taylor $\gamma_{e}=1$ y $\gamma_{m}$ queda indefinido, mientras que para las cotas de HS $\gamma_{e}=\gamma_{c}=1$ independientemente de las simetrías cristalinas. Por otro lado, los exponentes $\gamma_{e}$ y $\gamma_{m}$ para las cotas SC son diferentes y dependen de la simetría cristalina. Estos exponentes pueden expresarse en términos del número $J$ de sistemas de deslizamiento linealmente independientes en el límite $M \rightarrow \infty$. De los resultados numéricos se infiere que:

$$
\gamma_{e}=(4-J) / 2 \quad \text { y } \quad \gamma_{m}=(4-J)(3-J) / 2 .
$$

Esta expresión para $\gamma_{e}$ coincide con la inferida previamente por Nebozhyn et al. (2001) para la resistencia plástica al corte en policristales sin porosidad. En efecto, los resultados para las cotas $\mathrm{SC}$ mostrados en la figura 4.2a-b para sistemas de deslizamiento dominantes tipo $B(J=3)$ y en la figura $4.2 \mathrm{c}-\mathrm{d}$ para sistemas de deslizamiento dominantes tipo $A(J=2)$ son consistentes con los exponentes $\gamma_{e}=1 / 2 \mid \gamma_{m}=0$ y $\gamma_{e}=1$ | $\gamma_{m}=1$, respectivamente; mientras que aquellos mostrados en la figura 4.4a-b para sistemas de deslizamiento dominantes tipo basales + prismáticos $(J=4)$ y en la figura 4.4c-d para sistemas de deslizamiento dominantes tipo basales $(J=2)$ son consistentes con los exponentes $\gamma_{e}=0 \mid \gamma_{m}=0$ y $\gamma_{e}=1 \mid \gamma_{m}=1$, respectivamente. De acuerdo a estas leyes, puede haber agregados de cristales deficientes con porosidad que aún pueden acomodar deformaciones macroscópicas arbitrarias. Sin embargo, las deformaciones esféricas arbitrarias no pueden ser acomodadas por policristales con menos de tres sistemas independientes, mientras que deformaciones desviadoras no pueden ser acomodadas por policristales con menos de cuatro sistemas independientes. En otras palabras, los resultados SC aseguran que agregados cristalinos con porosidad y cuatro sistemas independientes pueden acomodar deformaciones arbitrarias, agregados de cristales con tres sistemas independientes pueden dilatar pero no distorsionarse y agregados de cristales con dos sistemas independientes no pueden deformarse. 


\subsection{Cotas de resistencia hidrostática}

En esta sección se presentan los resultados de las cotas de resistencia plástica hidrostática obtenidos en el capítulo anterior para distintos sólidos cristalinos. Las figuras 4.5 a 4.7 muestran las gráficas para varias cotas de resistencia hidrostática, como función de la porosidad, correspondientes a materiales con cinco sistemas de deslizamiento linealmente independientes $(J=5)$ : sólidos BCC e IONIC; sólidos FCC y sólidos HCP; sólidos IONIC con sistemas deficientes ( cinco sistemas de deslizamiento linealmente independientes $(J<5)$ : sólidos IONIC sin las familias A y C; HCP sin la familia C en la figura 4.8 -(a) y -(b) respectivamente.

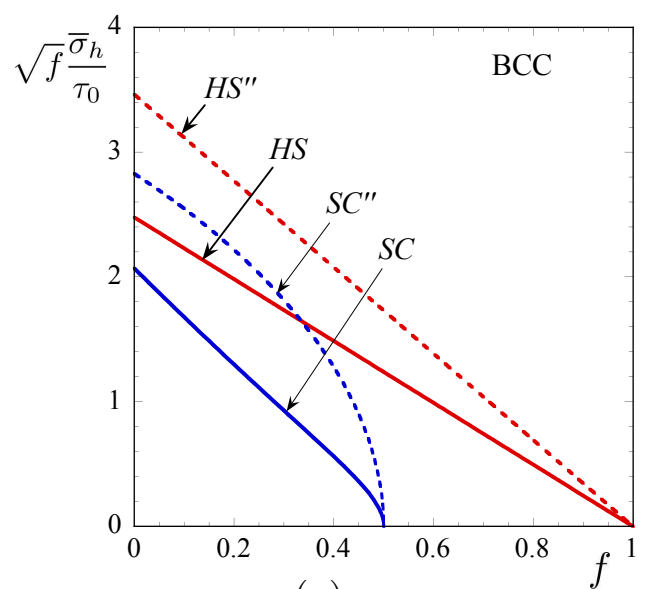

(a)

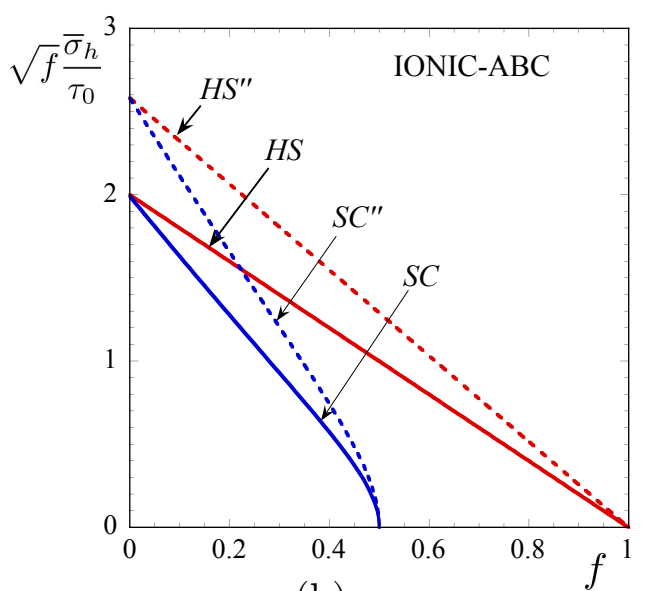

(b)

Figura 4.5: Cotas lineales de comparación del tipo Hashin-Shtrikman (HS) y Autoconsistentes (SC) para la resistencia plástica hidrostática de sólidos policristalinos porosos como función de la porosidad $f$ para materiales: (a) BCC, (b) IONIC. Las letras sin primar y doblemente primadas corresponden a cotas no relajadas y completamente relajadas respectivamente.

Recordemos que las cotas elementales de Taylor y Reuss resultan triviales -infinito e idénticamente nula respectivamente - en el contexto presente. Por otro lado, las cotas de de resistencia hidrostática presentadas anteriormente son todas finitas excepto en la 


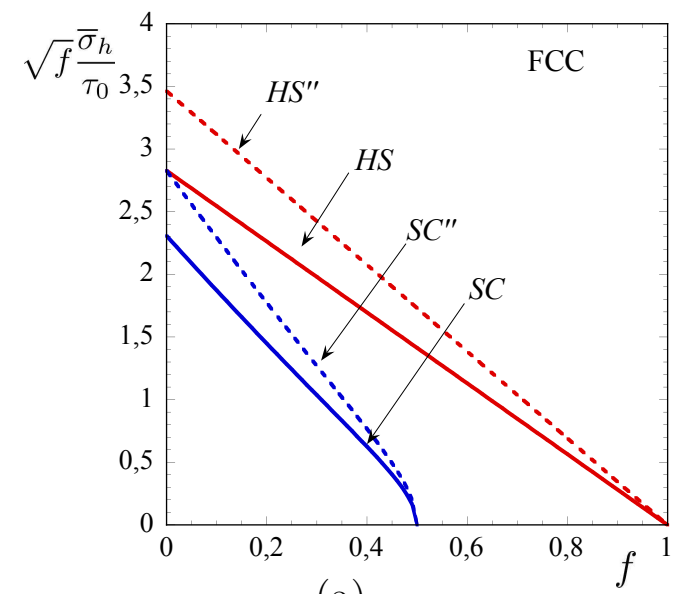

(a)

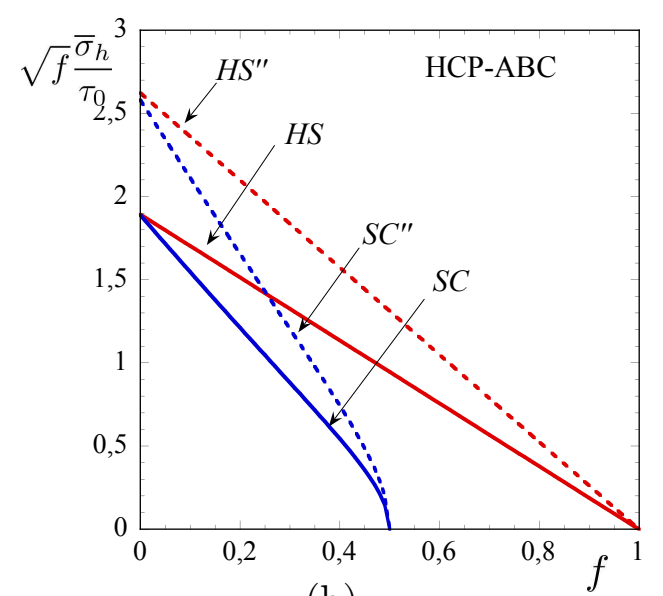

(b)

Figura 4.6: Cotas lineales de comparación del tipo Hashin-Shtrikman (HS) y Autoconsistentes (SC) para la resistencia plástica hidrostática de sólidos policristalinos porosos como función de la porosidad $f$ para materiales: (a) FCC, (b) HCP. Las letras sin primar y doblemente primadas corresponden a cotas no relajadas y completamente relajadas respectivamente.

percolación, en el límite diluido y en el caso de HS para policristales con sistemas linealmente independientes menores que cinco (es decir, $J<5$, véase figura 4.8). Las cotas SC toman valores finitos para porosidades $f<0,5$ en todos los casos. Los umbrales de percolación en HS y SC resultan $f=1$ y $f=0,5$ respectivamente. En el límite diluído el policristal se vuelve incompresible y la resistencia hidrostática tiende a infinito razón por la cual en las figuras 4.5 a 4.8 la resistencia hidrostática se ha multiplicado por una potencia de $f$ adecuada para visualizar el impacto de la relajación en las distintas cotas. 


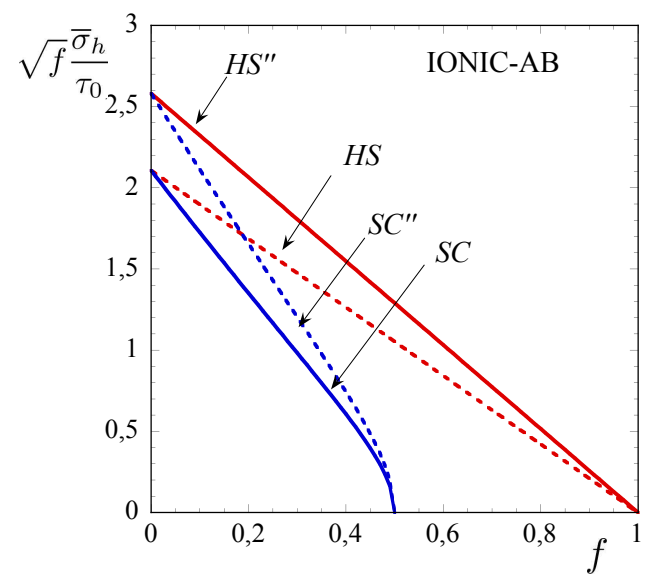

Figura 4.7: Cotas lineales de comparación del tipo Hashin-Shtrikman (HS) y Autoconsistentes (SC) para la resistencia plástica hidrostática de sólidos policristalinos porosos como función de la porosidad $f$ para materiales iónicos (IONIC) con sistemas deficientes (sin la familia $\mathrm{C}$ ). Las letras sin primar y doblemente primadas corresponden a cotas no relajadas y completamente relajadas respectivamente.

En todos los casos se observa que la relajación variacional tiene un impacto aumentando las cotas consideradas. Este impacto es independiente del nivel de porosidad resultando: para los materiales FCC y HCP sin la familia C ( 22\%); para sólidos IONIC sin las familias A y C $(\sim 23 \%)$; para IONIC $(\sim 30 \%)$; para sólidos BCC $(\sim 33 \%)$ y como era de esperarse el impacto máximo en sólidos $\mathrm{HCP}(\sim 37 \%)^{3}$. En cualquier caso, estos porcentajes son significantemente mayores que los presentados previamente por Idiart (2012) en el contexto de sólidos policristalinos sin porosidad $(<15 \%)$. Además, la relajación parece reducir la sensibilidad de las cotas a la cristalinidad de la matriz: las cotas relajadas no distinguen entre materiales FCC y BCC, véase ecuaciones (3.44) y (3.45); entre IONIC, IONIC sin la familia C y HCP, véase ecuaciones (3.46), (3.48) y (3.47). Los resultados SC también predicen un cambio sustancial en el juego entre la porosidad y la cristalinidad cuando los cristales son deficientes. De hecho, cuando los cristales no son deficientes, las cotas para la resistencia hidrostática siguen una ley de escalamiento diluida $\bar{\sigma}_{m}^{c} \sim f^{-1 / 2}$ (véase el Anexo A). Mientras que las cotas para sistemas

\footnotetext{
${ }^{3}$ Estos porcentajes corresponden a la diferencia entre las versiones no relajadas y completamente relajadas para la resistencia hidrostática relativa relativas a la cota no relajada.
} 


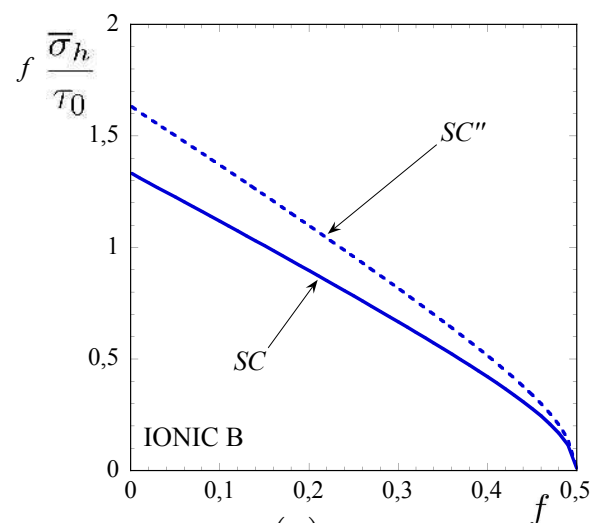

(a)

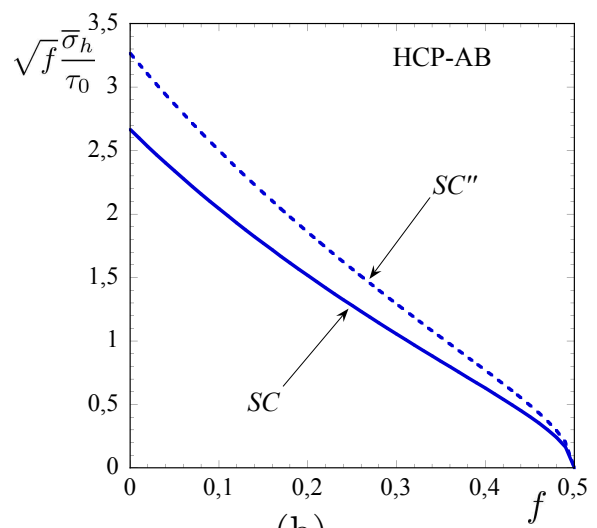

(b)

Figura 4.8: Cotas lineales de comparación del tipo Autoconsistentes (SC) para la resistencia plástica hidrostática de sólidos policristalinos porosos como función de la porosidad $f$ para materiales: (a) IONIC sin las familias A y C, (b) HCP sin la familia C. Las letras sin primar y doblemente primadas corresponden a cotas no relajadas y completamente relajadas respectivamente.

hexagonales que experimentan deslizamiento en los sistemas basal + prismático $(J=4)$ también siguen esta ley de escalamiento diluida, las cotas para sistemas IONIC que experimentan deslizamiento del tipo B $(J=3)$ siguen una ley de escalamiento diluida más débil $\bar{\sigma}_{m}^{c} \sim f^{-1}$. Un cambio en las leyes de escalamiento diluidas con las propiedades materiales o microestructurales es asociado típicamente con cambios en los patrones de localización que experimentan los campos de tasa de deformación. Es conocido que las técnicas de comparación lineal tienen la capacidad de capturar ciertos efectos de la localización de la tasa de deformación en la respuesta macroscópica de sólidos isótropos perfectamente plásticos (por ejemplo, Idiart \& Ponte Castañeda 2005, Idiart et al. 2006). Desde el punto de vista matemático, esta capacidad radica del uso de tensores de flexibilidad de comparación extremadamente anisótropos lo que permite la localización de campos de tasa de deformación en el medio lineal de comparación (véase Idiart et al. 2009). Esto es en verdad lo que produce un cambio en las leyes de escalamiento diluidas con la cristalinidad. De cualquier modo, la capacidad de capturar dichos cambios, en forma aproximada, es una propiedad destacable de las técnicas de comparación lineal la cual es confirmada ahora en un contexto más general. 


\section{Capítulo 5}

\section{Conclusiones de la tesis}

En este trabajo se derivaron cotas para la superficie de fluencia de sólidos policristalinos con porosidad presurizada mediante el método del medio lineal de comparación derivado en Idiart \& Ponte Castañeda (2007a, b). Se consideraron cotas para policristales porosos con textura isótropa y se consideraron distintas estructuras cristalinas: FCC, BCC, IONIC y HCP. Se consideró además el caso de policristales con sistemas deficientes para evaluar la influencia del contraste constitutivo. Por último se calculó en forma analítica la resistencia hidrostática en función de la porosidad dando cuenta de su impacto en las cotas. De esta forma se evaluaron las distintas formulaciones para las cotas relajadas y no relajadas con los distintos métodos de homogeneización - HS y SC- dando cuenta de la interrelación entre la porosidad, la cristalinidad y el contraste entre fases. Las principales conclusiones se enumeran a continuación:

- El efecto de la presión interna de los poros en la superficie de fluencia se tiene en cuenta con un simple cambio de variables.

- Las cotas no relajadas de Idiart \& Ponte Castañeda (2007) muestran mejoras respecto de las cotas relajadas de deBotton \& Ponte Castañeda (1995). Ésta puede ser tanto como $40 \%$ en el caso de policristales cúbicos y hexagonales. El grado de relajación depende de la cristalinidad de la matríz.

- Las cotas no relajadas de HS pueden ser más estrictas que las relajadas SC en determinadas estructuras cristalinas y por debajo de cierto nivel de porosidad.

- Se establecieron leyes de escalamiento para policristales con sistemas deficientes: 
analíticamente para la resistencia hidrostática y numéricamente para la resistencia al corte.

- De los análisis de resistencia hidrostática y al corte para sistemas policristalinos deficientes, se puede concluir que: policristales porosos con menos de cinco sistemas de deslizamientos independientes aún pueden acomodar deformaciones macroscópicas arbitrarias; las deformaciones esféricas arbitrarias no pueden ser acomodadas por policristales con menos de tres sistemas de deslizamientos linealmente independientes; las deformaciones desviadoras no pueden ser acomodadas por policristales con menos de cuatro sistemas de deslizamientos linealmente independientes.

- Las leyes de escalamiento diluída muestran, según la cristalinidad y el contraste, un cambio en el exponente de la porosidad. Esto es típico de la localización del campo de tensiones.

Las observaciones precedentes pueden ser aprovechadas en los esfuerzos actuales para desarrollar teorías policristalinas de plasticidad dilatacional a través de técnicas de comparación lineal. Estas técnicas son típicamente usadas en conjunto con la aproximación lineal SC, la cual es bien conocido que brinda estimaciones precisas para sólidos policristalinos lineales. Por otra parte, el uso de la aproximación lineal de las cotas de HashinShtrikman es descartado porque presumiblemente arroja cotas excesivamente rígidas. Si bien este es el caso en sólidos no porosos, los resultados anteriores muestran que puede no ser el caso en el contexto de policristales con bajo nivel de porosidad, el cual es precisamente el rango de aplicaciones ingenieriles como el estudio de la falla dúctil en metales y procesos de conformado. El uso de las aproximaciones de Hashin-Shtrikman en vez de las SC puede brindar ventajas desde el punto de vista práctico en vistas de reducir el costo computacional de la aproximación de Hashin-Shtrikman. La elección dependerá, sin embargo, del desempeño de las aproximaciones para respuestas constitutivas más generales (viscoplásticas) y condiciones de carga (no esféricas). En este sentido, recordemos que es conocido que las estimaciones arrojadas por el método del medio lineal de comparación para sistemas porosos son precisas para triaxialidades de tensiones bajas a moderadas pero son poco realistas a altas triaxialidades (Lebensohn et all 2011). Mientras que las 
mejoras encontradas en este trabajo no se espera que brinden estimaciones realistas para cargas hidrostáticas, se espera que aumenten el rango de triaxialidades para el cual las estimaciones son precisas.

Los programas experimentales desarrollados para evaluar el comportamiento de materiales nucleares son complejos por su naturaleza multivariable y muy costosos porque implican planes de irradiación que representan condiciones de operación normales, incidentales y hasta accidentales. Los resultados de estos experimentos se utilizan, por ejemplo, para comprender tanto la fenomenología de la evolución microestructural bajo irradiación como el comportamiento fenomenológico a escalas macroscópicas. Con esta información se calibran modelos multifísicos simples en un rango de variación de las variables fundamentales. Los modelos micromecánicos — como el desarrollado en este trabajo - se diseñan bajo hipótesis adecuadas para mejorar la comprensión de las desviaciones de los modelos multifísicos macroscópicos respecto de los experimentos. También se implementan en los modelos macroscópicos para ampliar el rango de ajuste de sus variables a las observaciones experimentales. Además se utilizan para extrapolar el comportamiento de los modelos macroscópicos a condiciones fuera del rango de aplicación de los experimentos generando nuevas estimaciones como, por ejemplo, en situaciones de variación de constantes características intrínsecas del material producto de variaciones microestructurales. A su vez los modelos micromecánicos se utilizan en el diseño de materiales para estimar su rango de comportamiento en función de variables microscópicas como la textura.

Por último, el modelo presentado en este trabajo puede ser adaptado a situaciones más realistas incorporando termoelasticidad, endurecimiento y viscoplasticidad para exponentes grandes partiendo de la solución de plasticidad perfecta como valor inicial. También puede incorporarse twinning como mecanismo de deformación agregando un término lineal al potencial del medio lineal de comparación. 



\section{Apéndice A}

\section{Aspectos numéricos}

\section{Notación de Woo}

Para la representación de los tensores de cuarto orden en el espacio de seis o cinco dimensiones se han utilizado la siguientes definiciones de la base de Woo introducidos en Woo (1985):

$$
\begin{aligned}
\mathbf{u}^{1} & =\frac{1}{\sqrt{3}}\left(\mathbf{e}_{1} \otimes \mathbf{e}_{1}+\mathbf{e}_{2} \otimes \mathbf{e}_{2}+\mathbf{e}_{3} \otimes \mathbf{e}_{3}\right) \\
\mathbf{u}^{2} & =\frac{1}{\sqrt{6}}\left(2 \mathbf{e}_{3} \otimes \mathbf{e}_{3}-\mathbf{e}_{1} \otimes \mathbf{e}_{1}-\mathbf{e}_{2} \otimes \mathbf{e}_{2}\right) \\
\mathbf{u}^{3} & =\frac{1}{\sqrt{2}}\left(\mathbf{e}_{1} \otimes \mathbf{e}_{1}-\mathbf{e}_{2} \otimes \mathbf{e}_{2}\right) \\
\mathbf{u}^{4} & =\frac{1}{\sqrt{2}}\left(\mathbf{e}_{2} \otimes \mathbf{e}_{3}+\mathbf{e}_{3} \otimes \mathbf{e}_{2}\right) \\
\mathbf{u}^{5} & =\frac{1}{2}\left(\mathbf{e}_{1} \otimes \mathbf{e}_{3}+\mathbf{e}_{3} \otimes \mathbf{e}_{1}\right) \\
\mathbf{u}^{6} & =\frac{1}{\sqrt{2}}\left(\mathbf{e}_{1} \otimes \mathbf{e}_{2}+\mathbf{e}_{2} \otimes \mathbf{e}_{1}\right)
\end{aligned}
$$

\section{Cotas de resistencia hidrostática: casos diluídos}

En el caso de policristales con sistemas deficientes, HCP AB sin la familia C $(J=4)$ y IONIC B sin las familias A y B $(J=3)$, si tomamos el límite cuando $f \rightarrow 0$ en las 
funciones objetivo se obtienen las siguientes expresiones.

$$
\begin{array}{ll}
\text { HCP AB: } g(f \rightarrow 0)= \begin{cases}\frac{4}{\sqrt{f}} \sqrt{2 / 3} & \mathrm{SC}^{\prime \prime} \\
\frac{1}{\sqrt{f}} \frac{3 \sqrt{3}}{2} & \mathrm{SC}\end{cases} \\
\text { IONIC B: } g(f \rightarrow 0)= \begin{cases}\frac{2 \sqrt{2 / 3}}{f} & \mathrm{SC}^{\prime \prime} \\
\frac{4}{3 f} & \mathrm{SC}\end{cases}
\end{array}
$$

\section{Tensores vértice $\hat{\boldsymbol{\sigma}}_{(\mathrm{v})}$}

Las funciones $v^{(r)}$ definidas por (3.14) requieren el conocimiento de los tensores vértice $\hat{\boldsymbol{\sigma}}_{(\mathrm{v})}$. Estos son los vértices de un polihedro cerrado en el espacio de tensiones de cincodimensiones cuyas caras son los $2 K$ planos definidos por $\boldsymbol{\sigma} \cdot \boldsymbol{\mu}_{(k)}= \pm \tau_{0}^{(k)}(k=1, \ldots, K)$. Para determinar el conjunto entero de vértices para un tipo de cristal particular se debe emplear un algoritmo de enumeración de vértices. Un algoritmo utilizado en la bibliografía está disponible en el trabajo de Avis \& Fukuda (1992). Sin embargo, encontramos más confiable emplear un algoritmo recientemente desarrollado por Terzer (2009) para enumerar rayos extremos de conos poliédricos multidimensionales. En esta metodología, se construye un cono poliédrico de seis dimensiones cuya sección transversal a una dada distancia del vértice forma la superficie de fluencia poliédrica de cinco dimensiones de un cristal simple. Luego la intersección entre los extremos de los rayos del cono y una sección transversal particular nos da los vértices de la superficie de fluencia.

\section{Tensor de flexibilidad efectivo $\widetilde{\mathbb{S}}$}

El potencial de tensiones efectivo $\widetilde{u}_{0}$ dado por (3.16) requiere el cálculo del tensor efectivo de flexibilidad $\widetilde{\mathbb{S}}$ dado por (3.17). La elección (3.18) y para el caso de texturas estadísticamente isótropas $\mathbb{Q}_{0}=4 \mu_{0} \mathbb{I}+\left(6 \mu_{0} / 5\right) \mathbb{K}$ —ver, por ejemplo, Willis (1982)— considerando la expresión (3.17) resulta $\widetilde{\mathbb{S}}$ explícito. Aquí, $\mathbb{J}$ y $\mathbb{K}$ son los tensores de proyección de corte e hidrostáticos de cuarto orden estándar e isótropos, respectivamente. 
Para la elección (3.19), el tensor $\mathbb{Q}_{0}$ depende de $\widetilde{\mathbb{S}}$ y la expresión (3.17) para $\widetilde{\mathbb{S}}$ es implícita. En el caso de tensión uniaxial, el tensor $\widetilde{\mathbb{S}}$ exhibe isotropía transversa alrededor de los ejes de tracción, y el tensor $\mathbb{Q}_{0}$ en términos de $\widetilde{\mathbb{S}}$ se puede calcular como se muestra - por ejemplo- en Willis (1982). La ecuación resultante (3.17) puede ser fácilmente resuelta por $\widetilde{\mathbb{S}}$ por el método del punto fijo.

\section{Procedimiento de optimización}

La optimización restringida respecto del tensor de flexibilidad simétrico, positivosemidefinido $\mathbb{S}^{(r)}$ en la cota (3.15) fue reescrita como un problema de optimización sin restricciones con respecto a los tensores de cuarto orden simétricos $\mathbb{A}^{(r)}$ tal que $\mathbb{S}^{(r)}=$ $\mathbb{A}^{(r)} \mathbb{A}^{(r)}$. Del mismo modo, la optimización restringida con respecto a las flexibilidades de deslizamiento positivas $\alpha_{(k)}^{(r)}$ en las cotas completamente relajadas, se reescribieron como una optimización sin restricciones con respecto a las variables escalares $a_{(k)}^{(r)}$ tal que $\alpha_{(k)}^{(r)}=\left(a_{(k)}^{(r)}\right)^{2}$. Luego las funciones objetivo fueron optimizadas utilizando el algoritmo de Brent (1973) para minimización sin derivadas. Este algoritmo requiere un valor inicial para las variables de optimización. Se siguió la siguiente secuencia:

1. cálculo de cotas completamente relajadas

1.1 con $\widetilde{\mathbb{S}}$ dado por la cota de Taylor y el valor inicial dado por $\alpha_{(k)}^{(r)}=\left(\tau_{0}^{(k)}\right)^{-1}$;

1.2 con $\widetilde{\mathbb{S}}$ dado por la cota de Hashin-Shtrikman y el valor inicial dado por el $\alpha_{(k)}^{(r)}$ óptimo del paso anterior;

1.3 con $\widetilde{\mathbb{S}}$ dado por la cota Autoconsistente y el valor inicial dado por el $\alpha_{(k)}^{(r)}$ óptimo del paso anterior;

2. cálculo de cotas relajadas

2.1 con $\widetilde{\mathbb{S}}$ dado por la cota de Hashin-Shtrikman y el valor inicial dado por el $\alpha_{(k)}^{(r)}$ óptimo del paso 1.2

2.2 con $\widetilde{\mathbb{S}}$ dado por la cota Autoconsistente y el valor inicial dado por el $\alpha_{(k)}^{(r)}$ óptimo del paso 1.3;

3. cálculo de cotas no relajadas

3.1 con $\widetilde{\mathbb{S}}$ dado por la cota de Hashin-Shtrikman y el valor inicial dado por el $\mathbb{S}^{(r)}$ óptimo del paso 2.1 ; 
3.2 con $\widetilde{\mathbb{S}}$ dado por la cota Autoconsistente y el valor inicial dado por el $\mathbb{S}^{(r)}$ óptimo del paso 2.2

En cada caso, el proceso de optimización fue detenido cuando el criterio de convergencia se satisface o el número de evaluaciones de la función alcanza $4 \times 10^{6}$. 
Apéndice B

\section{Cotas de resistencia hidrostática}

para sólidos policristalinos con

\section{sistemas deficientes}

En este apéndice se evalúa la resistencia hidrostática de policristales con sistemas de deslizamiento deficientes: cúbicos IONIC $\mathrm{AB}$ sin la familia $\mathrm{C}(\mathrm{J}=5)$ y con sistemas linealmente independientes menores a cinco: policristales hexagonales con familias $\mathrm{AB}$ sin la familia C $(\mathrm{J}=4)$ y IONIC B sin las familias A y B $(\mathrm{J}=3)$. Las ecuaciones (3.23) a (3.26) siguen siendo válidas mientras que el tensor óptimo $\widehat{\mathbb{S}}$ toma la forma de la simetría cristalina específica.

\section{Cotas no relajadas}

\section{Policristales IONIC AB sin la familia C}

El tensor de flexibilidad toma la forma:

$$
\hat{\mathbb{S}}=\frac{1}{2 \mu_{a}} \mathbb{K}_{a}+\frac{1}{2 \mu_{s}} \mathbb{K}_{s}=\frac{1}{2 \mu_{s}}\left[\mathbb{K}_{s}+\frac{1}{r} \mathbb{K}_{a}\right]
$$

donde $r \doteq \mu_{a} / \mu_{s}$ es la tasa de anisotropía cúbica y los tensores $\mathbb{K}_{a} \mathrm{y} \mathbb{K}_{s}$ como se definieron anteriormente. Para esta clase de tensores de flexibilidad, las funciones $\hat{v}$ pueden ser escritas respectivamente como 


$$
\hat{v}(\hat{\mathbb{S}})=\frac{\tau_{0}^{2}}{2 \mu_{s}} \operatorname{máx}_{\boldsymbol{\sigma} \in \hat{P}}\left\{\frac{1}{2}\left(\tau_{0}^{-1} \boldsymbol{\sigma}\right) \cdot\left[\mathbb{K}_{s}+\frac{1}{r} \mathbb{K}_{a}\right]\left(\tau_{0}^{-1} \boldsymbol{\sigma}\right)\right\} \doteq \frac{\tau_{0}^{2}}{2 \mu_{s}} \hat{v}(r),
$$

mientras que las prescripciones (3.26) para el módulo de referencia pueden ser escritas como $\mu_{0}=m_{0}(r, f) \mu_{s}$ donde debido a la simetría de la (B.1) con (3.27), $m_{0}(r, f)$ adopta la misma forma que la ecuación (3.31). Sin embargo cabe aclarar que los valores de $\mu_{a}$ y $\mu_{s}$ son propios de cada clase cristalina. Luego $\bar{\sigma}_{h}$ vendrá dado por la (3.32) donde la función objetivo será solución del problema da optimización (3.33).

\section{Policristales hexagonales AB}

El tensor de flexibilidad toma la forma:

$$
\hat{\mathbb{S}}=\left[\frac{1}{2 \mu_{h}} \mathbb{K}_{h}\right]
$$

donde el tensor $\mathbb{K}_{h}=\mathbb{K}_{c}+\mathbb{K}_{d}$. Nuevamente, para esta clase de tensores de flexibilidad, las funciones $\hat{v}$ pueden ser escritas respectivamente como

$$
\hat{v}(\hat{\mathbb{S}})=\frac{\tau_{0}^{2}}{2 \mu_{h}} \operatorname{máx}_{\boldsymbol{\sigma} \in \hat{P}}\left\{\frac{1}{2}\left(\tau_{0}^{-1} \boldsymbol{\sigma}\right) \cdot \mathbb{K}_{h}\left(\tau_{0}^{-1} \boldsymbol{\sigma}\right)\right\} \doteq \frac{\tau_{0}^{2}}{2 \mu_{h}} \hat{v},
$$

mientras que las prescripciones (3.26) para el módulo de referencia pueden ser escritas como $\mu_{0}=m_{0}(f) \mu_{h}$ siendo

$$
m_{0}(f)= \begin{cases}1 & \text { HS } \\ \frac{1-2 f}{1+2 f} \frac{2+f}{(1-f / 3)} & \text { SC }\end{cases}
$$

Luego $\bar{\sigma}_{h}$ vendrá dado por

$$
\frac{\bar{\sigma}_{h}}{\tau_{0}}=\frac{2}{\sqrt{3}} \frac{1-f}{\sqrt{f}} \sqrt{g(f)}
$$

donde $g(f)$ será solución de

$$
g(f)=\inf _{r \geq 0}\left[m_{0}(f) \hat{v}\right]
$$

Nótese que el producto a optimizar $m_{0}(f) \hat{v}$ es de grado cero en $\mathrm{r}$ y por lo tanto $g(f)=$ $m_{0}(f) \hat{v}$. 


\section{Policristales IONIC B}

El tensor de flexibilidad toma la forma:

$$
\hat{\mathbb{S}}=\frac{1}{2 \mu_{s}} \mathbb{K}_{s}
$$

Para esta clase de tensores de flexibilidad, las funciones $\hat{v}$ pueden ser escritas respectivamente como

$$
\hat{v}(\hat{\mathbb{S}})=\frac{\tau_{0}^{2}}{2 \mu_{s}} \operatorname{máx}\left\{\frac{1}{\boldsymbol{\sigma} \in \hat{P}}\left(\tau_{0}^{-1} \boldsymbol{\sigma}\right) \cdot \mathbb{K}_{s}\left(\tau_{0}^{-1} \boldsymbol{\sigma}\right)\right\} \doteq \frac{\tau_{0}^{2}}{2 \mu_{s}} \hat{v},
$$

mientras que las prescripciones (3.26) para el módulo de referencia pueden ser escritas como $\mu_{0}=m_{0}(f) \mu_{s}$ siendo

$$
m_{0}(f)= \begin{cases}1 & \text { HS } \\ \frac{1-2 f}{f} \frac{2+f}{3-f} & \text { SC }\end{cases}
$$

Luego $\bar{\sigma}_{h}$ vendrá dado por la ecuación (B.6) donde nuevamente la función $g(f)$ está dado por (B.7).

\section{Cotas completamente relajadas}

Las tensiones hidrostáticas $\overline{\boldsymbol{\sigma}}_{h}$ que pertenecen a la superficie de fluencia acotada (3.22) están dadas por una expresión análoga a (3.23) con las funciones $v\left(\mathbb{S}^{(s)}\right)$ reemplazadas por $v^{\prime \prime}\left(\alpha_{(k)}^{(s)}\right)$. Una vez más, debido a la simetría esférica de la microestructura y de las cargas, el policristal lineal de comparación debe ser isótropo y los tensores de flexibilidad asociados con el conjunto de $\alpha_{(k)}^{(r)}$ óptimos deben ser todos $\mathbf{Q}^{(r)}$ rotaciones de un simple tensor de flexibilidad con la simetría adecuada. Las cotas de HS y SC pueden ser obtenidas por caminos análogos a aquellos de la sección previa; el resultado puede ser escrito como (3.32)

$$
g(f)=\inf _{\alpha_{(k)} \geq 0}\left[m_{0}\left(r_{i}, f\right) \hat{v}^{\prime \prime}\left(\alpha_{(k)}\right)\right]
$$

En esta expresión, $r_{i}$ representa las constantes de anisotropía correspondientes. Para policristales deficientes IONIC AB sin la familia C $m_{0}(r, f)$ es la misma función (3.31), $\hat{v}^{\prime \prime} \doteq 2 \mu_{s} v^{\prime \prime} / \tau_{0}^{2}$; para cristales con sistemas deficientes $\mathrm{HCP}$ AB sin la familia $\mathrm{C} m_{0}(f)$ 
es la misma función (B.5), $\hat{v}^{\prime \prime} \doteq 2 \mu_{h} v^{\prime \prime} / \tau_{0}^{2}$; para cristales con sistemas deficientes IONIC B sin las familias A y C, $m_{0}(f)$ es la misma función (B.10). Donde para cristales IONIC $\mathrm{AB}$ sin la familia $\mathrm{C}$ con sistemas deficientes $r$ y $\hat{v}^{\prime \prime}$ corresponden a la ecuación (3.35); para cristales con sistemas deficientes $\mathrm{HCP} \mathrm{AB}$ sin la familia $\mathrm{C}$

$$
\hat{v}^{\prime \prime}\left(\alpha_{(k)}\right)=\frac{2 \sum_{k=1}^{K} \alpha_{(k)}}{\sum_{k=1}^{K} \alpha_{(k)} \boldsymbol{\mu}_{(k)} \cdot \mathbb{K}_{h} \boldsymbol{\mu}_{(k)}} ;
$$

para cristales con sistemas deficientes IONIC B sin las familias A y C, $\hat{v}^{\prime \prime}$ responde a la 3.35. La estructura matemática de la función objetivo en (B.11) implica que las flexibilidades óptimas de deslizamiento $\alpha_{(k)}$ asociadas con todos los sistemas de deslizamiento con iguales proyecciones tomarán los mismos valores: $\boldsymbol{\mu}_{(k)} \cdot \mathbb{K}_{s} \boldsymbol{\mu}_{(k)}$ y $\boldsymbol{\mu}_{(k)} \cdot \mathbb{K}_{a} \boldsymbol{\mu}_{(k)}$ para el caso de los cúbicos, IONIC AB y IONIC B según corresponda; $\boldsymbol{\mu}_{(k)} \cdot \mathbb{K}_{b} \boldsymbol{\mu}_{(k)}, \boldsymbol{\mu}_{(k)} \cdot \mathbb{K}_{c} \boldsymbol{\mu}_{(k)}$ y $\boldsymbol{\mu}_{(k)} \cdot \mathbb{K}_{d} \boldsymbol{\mu}_{(k)}$ para el caso de los HCP; $\boldsymbol{\mu}_{(k)} \cdot \mathbb{K}_{h} \boldsymbol{\mu}_{(k)}$ para el caso de cristales con sistemas deficientes HCP AB. 


\section{Bibliografía}

[1] Avis, D. \& Fukuda, K. (1992) A pivoting algorithm for convex hulls and vertex enumeration of arrangements and polyhedra. Discrete and Computational Geometry 8, 295-313.

[2] Bache, M. R. \& Evans, W. J. (2001) Impact of texture on mechanical properties in an advanced titanium alloy. Mater. Sci. Engrg. A 319-321, 409-414.

[3] Blair, P. (2008) Modelling of fission gas behaviour in high burnup nuclear fuel. Tesis Doctoral. École polytechnique. Francia.

[4] Bouchitté, G. \& Suquet, P. (1991) Homogenization, plasticity and yield design. En: Composite media and homogenization theory (G. Dal Maso \& G. Dell'Antonio, eds.). Birkhäuser, Basel, 107-133.

[5] Caré, S. \& Zaoui, A. (1996) Cavitation at triple nodes in $\alpha$-zirconium polycrystals. Acta Mater. 44, 1323-1336.

[6] deBotton, G. \& Ponte Castañeda, P. (1995) Variational estimates for the creep behavior of polycrystals. Proc. $R$. Soc. Lond. A 448, 121-142.

[7] Dendievel, R., Bonnet, G. \& Willis, J.R. (1991) Bounds for the creep behaviour of polycrystalline materials. En: Dvorak, G.J. (Ed.), Inelastic Deformation of Composite Materials, Springer, New York, 175-192.

[8] Dherbey, F. L. (2002) Elevated temperature creep of polycrystalline uranium dioxide: from microscopic mechanisms to macroscopic behaviour, Acta Mater. 50, 1359-6454. 
[9] Dubuisson, P. (2011) Le gonflement des aciers austénitiques. Revue de Métallurgie 108, 33-37.

[10] Edwards, D.J., Garner, F.A., Bruemmer, S.M. \& Efsing, P. (2009) Nano-cavities observed in a 316SS PWR flux thimble tube, J. Nucl. Mater. 384, 249-255.

[11] Edwards, D.J., Simonen, E.P. \& Bruemmer, S.M. (2003a) Evolution of fine-scale defects in stainless steels neutron-irradiated at $275^{\circ}$ C. J. Nucl. Mater. 317, 13-31.

[12] Garnier, J. (2007) Déformation sous flux des aciers austénitiques des structures internes des réacteurs à eau pressurisée. Reporte de Tesis de l'Institut National Polytechnique de Grenoble, Francia

[13] Gray III, G.T., Bourne, N.K., Zocher, M.A., Maudlin, P.J. \& Millett, J.C.F. (2000) Influence of crystallographic anisotropy on the Hopkinson fracture "spallation" of zirconium. En M.D. Furnish, L.C. Chhabildas \& R.S. Hixson, editors, Shock Compression of Condensed Matter 1999, AIP Press, Woodbury, NY, Estados Unidos, 509-512.

[14] Hales, S. J. \& Hafley, R. A. (1998) Texture and anisotropy in Al-Li alloy 2195 plate and near-net-shape extrusions. Mater. Sci. Engrg. A 257, 153-164.

[15] Han, X., Besson, J., Forest, S., Tanguy, B. \& Bugat, S. (2013) A yield function for single crystals containing voids. Int. J. Solids Struct. 50, 2115-2131.

[16] Holt, R.A. \& Causey, A.R. (2004) Volume conservation during irradiation growth of Zr-2.5Nb. J. Nucl. Mater. 335, 529-533.

[17] Idiart, M. I. (2012) Bounding the plastic strength of polycrystalline solids by linearcomparison homogenization methods. Proc. R. Soc. Lond. A 468, 1136-1153.

[18] Idiart, M. I. \& Ponte Castañeda, P. (2005) Second-order estimates for nonlinear isotropic composites with spherical pores and rigid particles. C. R. Mecanique 333, $147-154$. 
[19] Idiart, M. I., Moulinec, H., Ponte Castañeda, P. \& Suquet, P. (2006). Macroscopic behavior and field fluctuations in viscoplastic composites: second-order estimates vs full-field simulations. J. Mech. Phys. Solids 54, 1029-1063.

[20] Idiart, M. I. \& Ponte Castañeda, P. (2007a) Variational principles and bounds for nonlinear composites with anisotropic phases. I. General results. Proc. R. Soc. Lond. A 463, 907-924.

[21] Idiart, M. I. \& Ponte Castañeda, P. (2007b) Variational principles and bounds for nonlinear composites with anisotropic phases. II. Crystalline materials. Proc. R. Soc. Lond. A 463, 925-943.

[22] Idiart, M. I., Willot, F., Pellegrini, Y.-P. \& Ponte Castañeda, P. (2009) Infinitecontrast periodic composites with strongly nonlinear behavior: effective-medium theory versus full-field simulations. Int. J. Solids Struct. 46, 3365-3382.

[23] Jitsukawa, S., Ioka, I. \& Hishinuma, A. (1999) Post-irradiation mechanical properties of austenitic alloys at temperatures below 703 K, J. Nucl. Mater. 271-272, 167-172.

[24] Julien, J. (2008) Modélisation multi-échelles du couplage physico-chimie-mecánique du comportement du combustible à haute température des réacteurs à eau sous pression. Tesis Doctoral. Université de Provence Aix Marseille I, Francia.

[25] Kocik, J. (2002) TEM and PAS study of neutron orradiated VVER-type RPC steels. J. Nucl. Mater. 303, 52-64.

[26] Kocks, U. F., Tomé, C. N. \& Wenk, H.-R. (1998) Texture and anisotropy. Cambridge, U.K.: Cambridge University Press.

[27] Lebensohn, R.A., Liu, Y. \& Ponte Castañeda, P. (2004) On the accuracy of the self-consistent aroximation for polycrystals: comparison with full-field numerical simulations. Acta Mat. 52, 5347-5361. Lebensohn, R.A., Liu, Y. \& Ponte Castañeda, P. (2004) On the accuracy of the self-consistent aroximation for polycrystals: comparison with full-field numerical simulations. Acta Mat. 52, 5347-5361. 
[28] Lebensohn, R.A., Idiart, M.I., Ponte Castañeda, P. \& Vincent, P.-G. (2011) Dilatational viscoplasticity of polycrystalline solids with intergranular cavities. Phil. Mag. 91, 3038-3067.

[29] Liu, Y. \& Ponte Castañeda, P. (2004) Homogenization estimates for the average behavior and field fluctuations in cubic and hexagonal viscoplastic polycrystals. J. Mech. Phys. Solids 52, 1175-1211.

[30] Liu, Y., Gilormini, P. \& Ponte Castañeda, P. (2003) Variational self-consistent estimates for texture evolution in viscoplastic polycrystals. Acta Mater. 51, 5425-5437.

[31] Millet, J. C. F., Whiteman, G., Bourne, N. K. \& Gray III, G. T. (2008) The role of anisotropy in the response of the titanium alloy Ti-6Al-4V to shock loading. $J . A$. Phys. 104, 073531.

[32] Maziasz, P.J. \& Mc Hargue, C.J. (1987) Microstructural evolution in annealed austenitic steels during neutron irradiation. Int. Mat. Reviews 32, 190-219.

[33] Maziasz, P.J. (1992) Temperature dependence of the dislocation microstructure of PCA austenitic stainless steel irradiated in ORR spectrally-tailored experiment, J. Nucl. Mater. 191-194, 701-705.

[34] Maziasz, P.J. (1993) Overview of microstructural evolution in neutron irradiated austenitic stainless steels. J. Nucl. Mater. 205, 118-145.

[35] Nebozhyn, M.V., Gilormini, P. \& Ponte Castañeda, P. (2000) Variational selfconsistent estimates for viscoplastic polycrystals with highly anisotropic grains. C.R. Acad. Sci. Paris Ser. II 328, 11-17.

[36] Nebozhyn, M.V., Gilormini, P. \& Ponte Castañeda, P. (2001) Variational selfconsistent estimates for cubic viscoplastic polycrystals: the effects of grain anisotropy and shape. J. Mech. Phys. Solids 49, 313-340.

[37] Nomoto, A. (2014) Understanding on the mechanisms of irradiation embrittlement of RPV steels and development of embrittlement correlation method. IAEA workshop. 
[38] Olander, D. R. (1976) Fundamental Aspects of Reactor Fuel Elements. TID-27611P1. Ed. Oak Ridge, Tenn.

[39] Pokor, C. (2003) Caractérisation microstructurale et modélisation du durcissement des aciers austénitiques irradiés des structures internes des réacteurs à eau pressurisée. Reporte de Tesis de l'Institut National Polytechnique de Grenoble, Raport CEA-R-6031, Francia.

[40] Rockafellar, T. (1970) Convex analysis. Princeton University Press, Princeton, U.S.A.

[41] Rodchenkov, B.S., Kalinin, G.M., Strebkov, Y.S., Shamardin, V.K., Prokhorov, V.I. \& Bulanova, T.M. (2009). Irradiation behavior of Ti-stabilized 316L type steel, J. Nucl. Mater. 386-388, 277-280.

[42] Rogge, R. \& Holt, R.A. (2006) Development of local microestructure and crystallographic texture in extruded Zr-2.5Nb tubes. Mater. Sci. Engrg. A 437, 10-20.

[43] Serrano, M. (2013). Workshop on improved RPV structural integrity assessment for Atucha NPP, IAEA, CAC, Buenos Aires, Argentina.

[44] Sobol, I.M. (1967) On the distribution of points in a cube and the aroximate evaluation of integrals. USSR Computat. Math. Math. Phys. 7, 86-112.

[45] Soneda, N. (2015) Irradiation Embrittlement of Reactor Pressure Vessels (RPVs) in Nuclear Power Plants. A volume in Woodhead Publishing Series in Energy. Ed. Elsevier [46] Suquet, P. (1983) Analyse limite et homogénéisation. C.R. Acad. Sci. Paris Ser. II 296, 1355-1358.

[47] Torimaru, T., Kodama, M., Tanaka, S., Nakamura, T., Kumagai, K. \& Asano, K. (2010) Fracture Toughness of Austenitic Stainless Steels after BWR Irradiation. Symposium Fontevraud 7 - Contribution of Materials Investigations to Improve the Safety and Performance of LWRs, Paper Reference $n^{\circ}$ A060-T02, Avignon, Francia, 26-30, 2010 . 
[48] Terzer, M. (2009) Large Scale Methods to Enumerate Extreme Rays and Elementary Modes. Tesis Doctoral, Swiss Federal Institute of Technology, Switzerland.

[49] Vincent, P.-G. (2007) Modélisation micromécanique de la croissance et de la percolation de pores sous pression dans una matrice céramique à haute tempèrature. Tesis Doctoral, Université de Provence Aix Marseille I, Marseille, France.

[50] Vincent, P.-G., Monerie, Y. \& Suquet, P. (2009) Porous materials with two populations of voids under internal pressure: I. Instantaneous constitutive relations. Int. J. Solids Struct. 46, 480-506.

[51] Walpole, L.J. (1981) Elastic behavior of composite materials: theoretical foundations. Adv. Apl. Mech. 21, 169-242.

[52] Willis, J.R. (1977) Bounds and self-consistent estimates for the overall moduli of anisotropic composites. J. Mech. Phys. Solids 25, 185-202.

[53] Willis, J.R. (1982) Elasticity theory of composites. En: Hopkins, H. G., Sewell, M. J. (Eds.), Mechanics of Solids, The Rodney Hill 60th Anniversary Volume. Pergamon Press, 653-686.

[54] Willis, J.R. (1994) Upper and lower bounds for nonlinear composite behavior. Mater. Sci. Engrg. A 175, 7-14.

[55] Woo, C.H. (1985) Polycrystalline effects on irradiationn creep and growth in textured zirconium. J. Nucl. Mater. 131, 105-117.

[56] Zinkle, S.J. \& Was, G.S. (2013) Materials challenges in nuclear energy, Acta Mater. 61, 735-758.

[57] Zhang, H. (2013) Irradiation induced damage in CANDU spacer material Inconel X-750. Tesis Doctoral, Queen's University, Kingston, Ontario, Canada. 